\title{
The evolution of HCG 31: Optical and high-resolution HI study ${ }^{\star}$
}

\author{
L. Verdes-Montenegro ${ }^{1}$, A. Del Olmo ${ }^{1}$, M. S. Yun ${ }^{2}$, and J. Perea ${ }^{1}$ \\ 1 Instituto de Astrofísica de Andalucía, CSIC, Apdo. 3004, 18080 Granada, Spain \\ e-mail: lourdes@iaa.es \\ 2 Department of Astronomy, University of Massachusetts, Amherst, MA 01003, USA
}

Received 15 January 2004 / Accepted 28 September 2004

\begin{abstract}
Here we present the results of our new optical imaging and spectroscopic study and the analysis of new highresolution HI images of the Hickson Compact Group HCG 31. Taking advantage of the improved sensitivity and angular resolution of the new optical and $\mathrm{HI}$ images, we have identified an extensive complex of stellar and HI tidal features and their kinematics. Our HI study show that H31A and C are not an advanced merger since their velocity fields can be still separated and have almost orthogonal orientations. All of the current sites of ongoing active star formation are shown to be associated with the highest column density peaks traced in HI. A new companion A0500-0434 located $240 \mathrm{kpc}$ south of the group center is also discovered in HI. A detailed scenario for the tidal interactions involved and the origins of the individual tidal features are constructed using the morphology and kinematics of the tidal features. The derived dynamical mass for the entire group is about $2 \times 10^{11} M_{\odot}$, which is a few times larger than the sum of the masses of the individual group galaxies. The ultimate fate of the group is that HCG 31 is probably on its way to form a single HI cloud group containing all galaxies.
\end{abstract}

Key words. galaxies: individual: HCG 31 - galaxies: interactions - galaxies: kinematics and dynamics - galaxies: evolution galaxies: structure - radio lines: galaxies

\section{Introduction}

Hickson compact groups (HCGs) are highly isolated, dense galaxy systems (Hickson et al. 1992), and therefore tidal interactions are expected to be continuous and dynamically important. For this reason they represent unique laboratories for studying interactions in extreme environments and tidally induced star formation as well as morphological and dynamical evolution of galaxies in general. This study is part of a broader investigation of the evolutionary status of HCGs. Analyzing the distribution and kinematics of HI emission in a sample of $16 \mathrm{HGCs,}$ we have already established a strong evolution of $\mathrm{HI}$ content of the groups and individual member galaxies, following a broad evolutionary scenario of increasing HI deficiency with the group evolution (Verdes-Montenegro et al. 2001, hereafter VM01). Our CO survey of a complete sample of HCGs have shown that about $20 \%$ of the galaxies are deficient in $\mathrm{CO}$ when compared with a sample of isolated field galaxies (Verdes-Montenegro et al. 1998). A detailed CO

* Based on observations made with the VLA operated by the National Radio Astronomy Observatory (the National Radio Astronomy Observatory is a facility of the National Science Foundation operated under cooperative agreement by Associated Universities, Inc.) and on data taken using ALFOSC, which is owned by the Instituto de Astrofísica de Andalucía (IAA) and operated at the Nordic Optical Telescope under agreement between IAA and the NBIfA of the Astronomical Observatory of Copenhagen. imaging study of HCG 31 and HCG 92 using the OVRO interferometer has revealed a highly perturbed distribution of the molecular gas (Yun et al. 1997). Gas stripping and exhaustion by tidally induced star formation were discussed as possible explanations for the CO deficiency. In this paper we are examining the proposed scenario for the nature and evolution of HCG 31 using new high spatial and spectral resolution HI mapping and optical data.

Four galaxies (H31A-D) were identified by Hickson (1982) in the Palomar Observatory Sky Survey (POSS) as the members of HCG 31. Whether H31A and H31C (Mk 1089) are two separate galaxies or a merger in its final stage has been the subject of frequent debates because of to their overlapping and irregular morphologies (see below). H31B is a small spiral close to H31A and H31C. H31D is a background galaxy with a redshift of $\sim 23000 \mathrm{~km} \mathrm{~s}^{-1}$ (Hickson et al. 1992). Three new emission line objects, H31E, F and G (H31G is also a Markarian galaxy, MK 1090) were identified by Rubin et al. (1990, hereafter R90). Rubin et al. also noted the presence of another small galaxy (H31Q or NPM16-04.0219 in NED ${ }^{1}$ ) with $m_{B}=16.24$ about $2^{\prime}$ north of H31A and H31C. Even though no redshift information was available, its membership to HCG 31 was suggested by R90 using its apparent association

\footnotetext{
1 The NASA/IPAC extragalactic database (NED) is operated by the Jet Propulsion Laboratory, California Institute of Technology, under contract with the National Aeronautics and Space Administration.
} 
within the HI cloud imaged using the VLA by Williams et al. (1991, hereafter W91). Richer et al. (2003) obtained the redshift of this galaxy, providing a further support for its membership to the group. The large scale kinematics of the atomic gas in the large HI cloud, uncorrelated with the individual galaxies, was interpreted as an indication of on-going merging process for the entire group by W91 and others. The group displays evidence of vigorous ongoing star formation including very young globular clusters formed $10 \mathrm{Myr}$ ago in a starburst episode induced by the interaction, and $\mathrm{H} 31 \mathrm{~F}$ appears to be the youngest galaxy of the group made of gas-rich material stripped from the A+C complex (Iglesias-Páramo \& Vílchez 1997; Johnson et al. 1999; Johnson \& Conti 2000; O’Halloran et al. 2002; Lopez-Sanchez et al. 2004).

In this paper we report a detailed study of HCG 31 based on new optical images obtained under good seeing conditions as well as new HI observations obtained using the VLA. Taking advantage of the improvements in the editing and calibration software and new imaging tools in AIPS, we were able to achieve a three fold improvement in the overall sensitivity of the W91 HI data (see Sect. 2) and reveal new details about the HI distribution in HCG 31. Further, new higher resolution observations are also made in the CnB configuration of the VLA, and various $\mathrm{HI}$ features are examined in greater detail. We report the detection of a new HI companion $15^{\prime}$ south of the group center.

The observations and data reduction are described in Sect. 2, and the group environment is examined in Sect. 3. We discuss our results for the individual galaxies in Sect. 4 and for the intergalactic material in Sect. 5. Throughout this paper, tidal features are referred to as "t-" plus a short identification (e.g. " $t-S$ " for the southern tidal tail). A global discussion of HCG 31 is presented in Sect. 6. We adopt a distance to HCG 31 of 54.3 Mpc assuming a Hubble constant $H_{0}=75 \mathrm{~km} \mathrm{~s}^{-1} \mathrm{Mpc}^{-1}$ and the derived mean heliocentric velocity of $4076 \mathrm{~km} \mathrm{~s}^{-1}$ (Sect. 3) ${ }^{1}$.

\section{Observations}

\subsection{Optical data}

We obtained deep CCD images of HCG 31 in the Johnson BVRI filters at the $1.5 \mathrm{~m}$ telescope of the Centro Astronómico Hispano-Alemán in Calar Alto (CAHA, Spain). These images with a field of view of $5: 4 \times 5: 4$ include the central galaxies $\mathrm{H} 31 \mathrm{~A}, \mathrm{H} 31 \mathrm{~B}$, and $\mathrm{H} 31 \mathrm{C}$, as well as $\mathrm{H} 31 \mathrm{G}$ to the south, 2 '4 from the center of the main group. An additional $B$-band image with a larger field of view $(6.5 \times 6.5)$ including H31Q, $2^{\prime}$ to the north, was taken at the $2.5 \mathrm{~m}$ Nordic Optical Telescope (NOT) in La Palma (Spain) with ALFOSC, and this image is used primarily for the description of the group (see Fig. 1). The galaxy A0500-0434, a new member discovered in our HI data (see Sect. 4.5), lies outside the imaged field, and a new $R$ image was obtained using the NOT. A summary of the observations is

\footnotetext{
1 The correction due to the Virgo flow is negligible since the flow direction $\left(l \sim 276^{\circ}, b \sim+30^{\circ}\right)$ is nearly perpendicular to the direction of HCG 31 .
}

given in Table 1. Atmospheric conditions were photometric and the seeing was between 1." 3 to $1 . " 5$ during these runs.

The reduction and calibration of the images were carried out using standard techniques. The atmospheric extinction was determined from observations of 11 standard stars from the Landolt list $(1983,1992)$. The flux calibration was carried out following the method developed by Young (1974) and the magnitudes of the standard stars of Landolt were transformed to the Johnson system for $R$ and $I$ filters through the relations given by Bessell $(1983,1995)$. Residuals in the standard stars in the final calibration were always smaller than 0.03 mag in all color indices. The errors due to variations in the sky were smaller than $1 \%$. Once the images were calibrated, surface brightness sensitivity achieved are 24, 24.5, 23.5 and $23 \mathrm{mag} / \operatorname{arcsec}^{2}$ in the CAHA $B, V, R$ and $I$-band respectively. The NOT $B$-band image has been smoothed by a median filtering of $4^{\prime \prime} \times 4^{\prime \prime}$ for a better sensitivity to extended structures, reaching a surface brightness of $26.6 \mathrm{mag} / \mathrm{arcsec}^{2}$. Color indices given in this paper have been corrected for Galactic absorption using the values determined by Burstein \& Heiles (1984) and the reddening law from Savage \& Mathis (1979).

The $B$-band NOT image of the group is shown in Fig. 1, where we have labeled all the previously identified galaxies of the group (A, B, C, G and Q). A long optical tail runs from the south of H31A and H31C to H31G. In the area of knots E and F identified by R90 we find substructure consisting of small knots that we have labeled as e1 - e5 and $\mathrm{f} 1$ - f7 (see inset image in Fig. 1). Other weak knots are also found around H31Q (Sect. 4.4) and in the wide extended region at the north of $\mathrm{H} 31 \mathrm{~A}$ and $\mathrm{H} 31 \mathrm{C}$.

The $V$ and $R$-band images of the central group and the $B-I$ color index image of the central galaxies of the group are shown in Fig. 2. In order to enhance the small scale structures a sharpened $R$-band image was obtained by the subtraction of a $3^{\prime \prime} \times 3^{\prime \prime}$ median filtered image and it is shown in Fig. 2 d. Only the most intense found features are taken into account for the interpretation of the data, and its reality has been always checked in the original unsharpened image.

Long slit spectra of H31Q and NPM1G -04.0218 (see Sect. 3) were obtained at the NOT telescope using the ALFOSC spectrograph. Table 1 summarizes the spectra taken for this study The spectra were reduced using the standard methods. The rms error in the wavelength calibration was less than $0.1 \AA$.

\subsection{VLA HI data}

Two complementary sets of HI data are analyzed in this paper. New high resolution VLA HI data have been obtained in the CnB configuration in June 1997. HCG 31 was observed for 4 hours using 27 antennas with the phase center at $\alpha(1950)=04^{\mathrm{h}} 59^{\mathrm{m}} 09^{\mathrm{s}} .0, \delta(1950)=-04^{\circ} 19^{\prime} 42^{\prime \prime}$. A velocity range between $3704 \mathrm{~km} \mathrm{~s}^{-1}$ and $4360 \mathrm{~km} \mathrm{~s}^{-1}$ was covered with a $3.125 \mathrm{MHz}$ bandwidth in the 2IF mode, identical to W91, but with a factor two improvement in velocity resolution $\left(10.6 \mathrm{~km} \mathrm{~s}^{-1}\right)$. The data were calibrated following the standard procedures, and a self-calibration algorithm was used to 


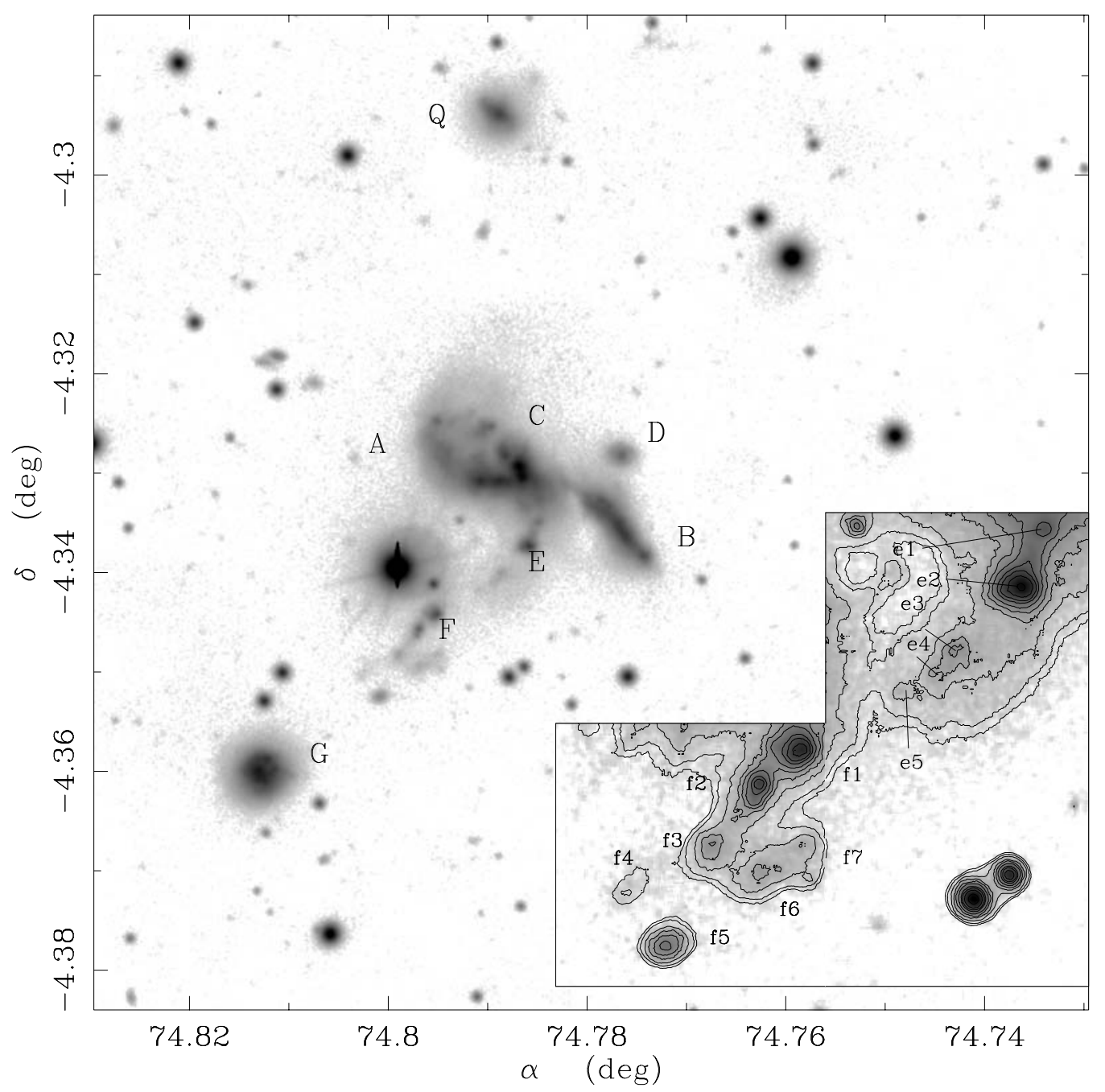

Fig. 1. $B$-band image of HCG 31 obtained at NOT, in a logarithmic gray-scale representation. Higher intensities are darker. It has been smoothed with a $4^{\prime \prime} \times 4^{\prime \prime}$ median filtering. The individual group members are identified as A, B, C, D, E, F, G, and Q. The area of objects E and F is enlarged in the lower right panel, and the bright emission knots further identified and labeled in the inset. The isophotal contours correspond to 20.9, 21.8, 22.4, 22.8, 23.4, 23.9, 24.4, 25.0, 25.5 and $25.7 \mathrm{mag} \operatorname{arcsec}^{-2}$. The orientation of all the images is North up and East to the left.

Table 1. Summary of photometric and spectroscopic observations.

\begin{tabular}{llclcccc}
\hline \hline Observations & Telescope & $\begin{array}{c}\text { Filter/ } \\
\Delta \lambda(\AA)\end{array}$ & Galaxies & $\begin{array}{c}\text { Spatial } \\
\text { scale }\left({ }^{\prime \prime} / \mathrm{px}\right)\end{array}$ & $\begin{array}{c}T_{\text {exp }} \\
(\mathrm{s})\end{array}$ & $\begin{array}{c}\text { Disper. } \\
(\AA / \mathrm{Ax})\end{array}$ & $\begin{array}{c}\text { Slit } \\
\left({ }^{\prime \prime}\right)\end{array}$ \\
\hline Broad-band & $1.5 \mathrm{~m}$ CAHA & $B$ & H31ABCGF & 0.32 & 2400 & - & - \\
Images & $1.5 \mathrm{~m}$ CAHA & $V$ & H31ABCGF & 0.32 & 1500 & - & - \\
& $1.5 \mathrm{~m}$ CAHA & $R$ & H31ABCGF & 0.32 & 1800 & - & - \\
& $1.5 \mathrm{~m}$ CAHA & $I$ & H31ABCGF & 0.32 & 1200 & - & - \\
& $2.5 \mathrm{~m}$ NOT & $B$ & H31ABCGFQ & 0.19 & 1500 & - & - \\
& $2.5 \mathrm{~m}$ NOT & $R$ & A0500-0434 & 0.19 & 600 & - & - \\
\hline Long-slit & $2.5 \mathrm{~m}$ NOT & $5820-8330$ & H31Q & 0.19 & 1800 & 1.23 & 1.2 \\
Spectroscopy & 2.5 m NOT & $3000-9000$ & NPM1G-040218 & 0.19 & 800 & 2.96 & 1.2 \\
\hline
\end{tabular}

improve the image fidelity. Natural weighting of the data gives a synthesized beam of $15^{\prime \prime} 8 \times 14$.' $^{\prime}$, and the resulting rms noise in each channel map is $0.46 \mathrm{mJy}$ beam $^{-1}(1.20 \mathrm{~K})$. At an assumed distance of $54.3 \mathrm{Mpc}$ and an $\mathrm{HI}$ line width of $30 \mathrm{~km} \mathrm{~s}^{-1}$, the achieved HI mass detection limit is $1 \times 10^{7} M_{\odot} /$ beam. We have re-processed the archival VLA HI data originally obtained by W91 in the DnC configuration. This data set suffers from a serious radio interference problem. After careful editing of the data using an improved software, we were able to achieve rms noise level of $0.58 \mathrm{mJy}^{-1}$ beam $^{-1}(0.89 \mathrm{~K}$ with a 


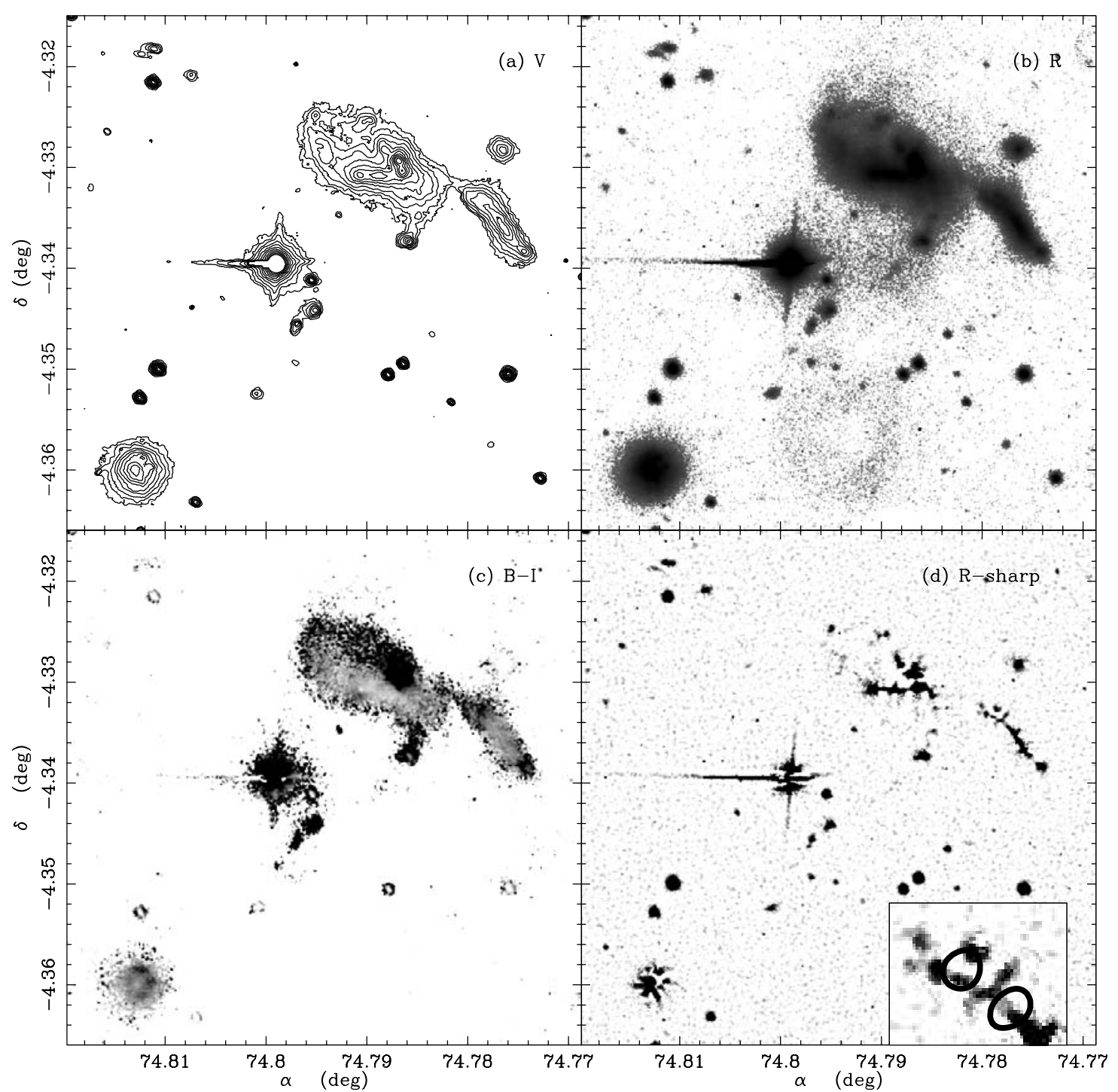

Fig. 2. a) Isophotal contours corresponding to the $V$ filter image, ranging from 18.5 to $23.5 \mathrm{mag} \operatorname{arcsec}^{-2}$, with a step of $0.5 \mathrm{mag} \operatorname{arcsec}{ }^{-2}$. b) $R$ image of the group represented as in a. c) $B-I$ color image in a gray-scale where black is bluer and white is redder. d) Sharpened $R$ image obtained by the subtraction of a $3^{\prime \prime} \times 3^{\prime \prime}$ median filtered image. In the lower right a zoom of the NE side of H31B is shown and the CO emission from Yun et al. (1997) is overplotted.

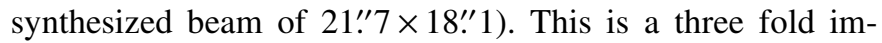
provement over the published images in W91, and additional low level extended emission features are recovered. The standard VLA calibration procedure is generally accurate enough to produce an absolute flux uncertainty of about $15 \%$.

The velocity integrated HI emission image obtained from our new reduction of the DnC configuration data is shown overlaid on the smoothed $B$-band image of HCG 31 in Fig. 3a. When compared with Fig. 7 of W91, the new HI map reveals new details such as the low level emission connecting the central cloud containing $\mathrm{H} 31 \mathrm{~A}, \mathrm{H} 31 \mathrm{~B}$, and $\mathrm{H} 31 \mathrm{C}$ with the northern emission west of H31Q. The velocity integrated HI image from our new $\mathrm{CnB}$ array data is shown in Fig. 3b. The central area shows a significant improvement due to the higher spatial resolution, and the extended emission to the east is also better resolved now. In addition, these new data have allowed us to separate the $\mathrm{HI}$ emission of $\mathrm{H} 31 \mathrm{~A}$ and $\mathrm{C}$ and to distinguish their kinematics (see Sect. 3) as well as to resolve the kinematics of H31F (Sect. 5.3). The emission that connects the northeastern side of the central cloud and H31Q was previously undetected, and this suggests this feature is clumpy. Some of the extended emission seen in the lower resolution map (Fig. 3a) is not seen in the new $\mathrm{CnB}$ configuration image, and some of the HI emission is quite diffuse and smoothly distributed. The individual tidal features discussed through out this paper are identified in this figure. The intensity weighted mean radial velocity field derived from the re-processed $\mathrm{DnC}$ and new $\mathrm{CnB}$ array data are plotted respectively in Figs. $3 \mathrm{c}$ and $3 \mathrm{~d}$. Our global velocity maps are consistent with the one obtained by Richer et al. (2003) from $\mathrm{H} \alpha$ data for $\mathrm{H} 31 \mathrm{AC}, \mathrm{B}$ and G. Further, our new data can distinguish and separate the velocity field in H31A and $\mathrm{H} 31 \mathrm{C}$. This is consistent with their velocity dispersion map of $\mathrm{H} 31 \mathrm{AC}$ where the higher values are reached in the overlapping area of the two velocity components that we have found for $\mathrm{H} 31 \mathrm{~A}$ and $\mathrm{C}$ respectively. 


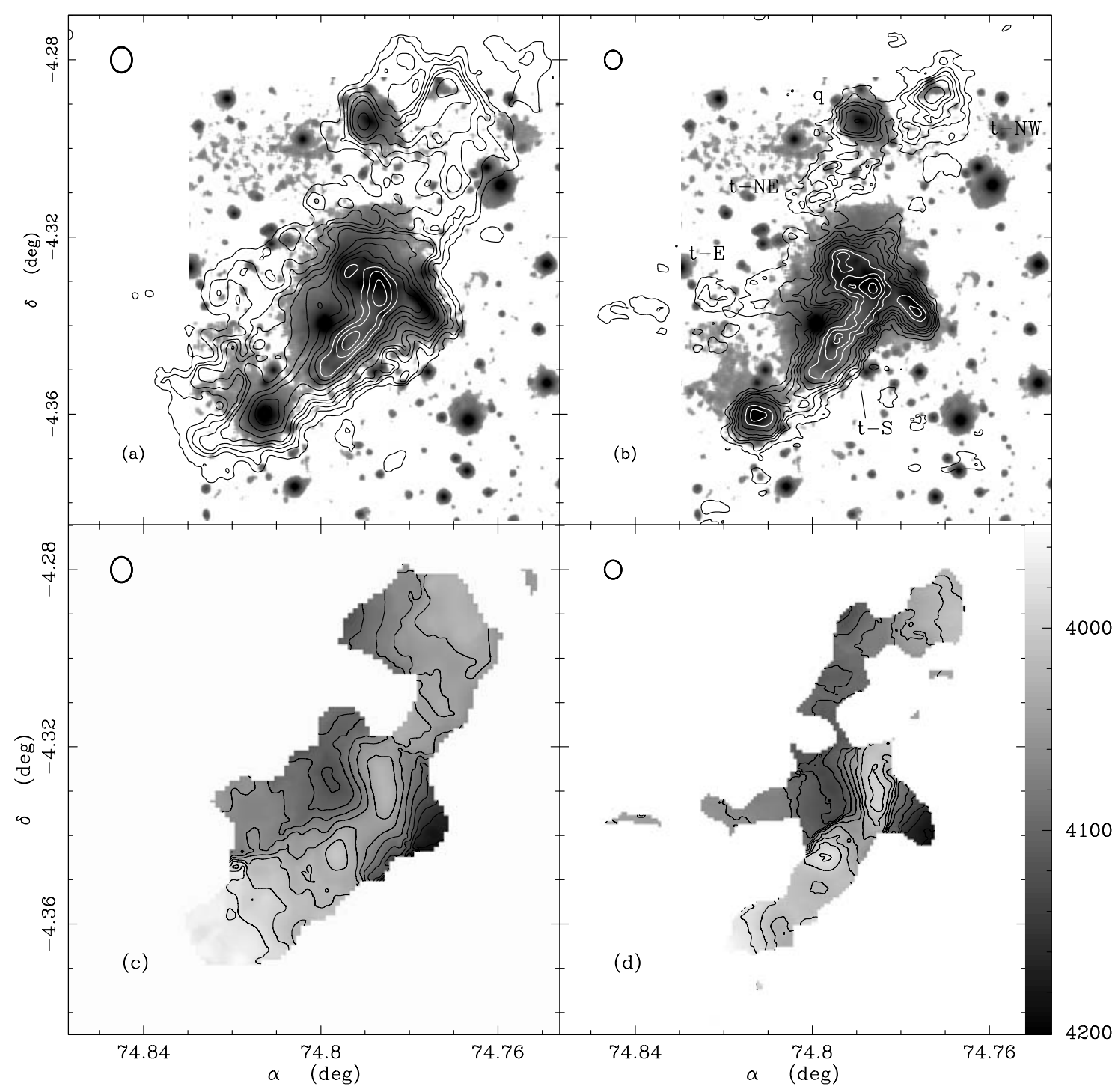

Fig. 3. a) Map of the HI column density distribution in HCG 31 obtained from a new reduction of the DnC configuration data, superimposed on a B image median smoothed by $4^{\prime \prime} \times 4^{\prime \prime}$. The contours correspond to column densities of 1.4, 2.8, 4.1, 5.5, 6.9, 10.3, 13.8, 17.2, 20.6, 24.1, 27.5 and $31.0 \times 10^{20}$ atoms $\mathrm{cm}^{-2}$. The beam size (shown on the upper right corner) is 21 .' $^{\prime} \times 18$.' $^{\prime} 1$. b) The same as a) for the new VLA $\mathrm{CnB}$ array data. The contours correspond to column densities of 1.0, 2.4, 3.8, 5.2, 6.9, 9.6, 13.0, 16.5, 19.9 and $23.3 \times 10^{20}$ atoms cm ${ }^{-2}$. The beam size is 15 ." $8 \times 14$." 5 . The four brightest tidal features are identified by "t-" plus a short identification according to their location. The highest contours are plotted in white for clarity. c) Map of the first moment of the HI radial velocity in HCG 31 obtained from a new reduction of the DnC configuration data shown both with iso-velocity contours and gray-scale. The contours range between 3980 and $4180 \mathrm{~km} \mathrm{~s} \mathrm{sith}^{-1}$ a step of $20 \mathrm{~km} \mathrm{~s}^{-1}$. The beam size is $21^{\prime \prime} 7 \times 18^{\prime \prime} 1$. d) The same as c) for the new CnB array data. The beam size is 15 ." $^{\circ} \times 14$." $^{\prime}$. The gray scale in panels c) and d) represents heliocentric velocities in $\mathrm{km} \mathrm{s}^{-1}$ as shown in the wedge.

The velocity channel maps for the high resolution $\mathrm{CnB}$ configuration VLA HI data are shown in Fig. 4 superposed on the $B$-band image.

The global HI spectra are derived by integrating the emission in the individual channel maps for both $\mathrm{DnC}$ and $\mathrm{CnB}$ configuration data and are shown in Fig. 5a using a thick and thin line respectively. We have also obtained spectra for individual galaxies and tidal features as shown in Figs. 5b-k. Identification of the corresponding velocity ranges has been often problematic due to source confusion. We have determined them from the complementary information in $\mathrm{DnC}$ and $\mathrm{CnB}$ array data. The higher resolution $\mathrm{CnB}$ data allow better distinction of overlapping features, but the Dnc data are required to confirm the reality of weak features in the $\mathrm{CnB}$ array data. The derived line parameters, velocities and total HI masses are summarized in Table 2 together with the detection ranges. The total $\mathrm{HI}$ line flux detected by the $\mathrm{DnC}$ array is $47 \%$ larger than the single dish data. The observing telescope beam (10'8) of Williams \& Rood (1987) was not large enough to include the emission from A0500-0434, but this alone cannot account for the entire difference because A0500-0434 contributes less than $5 \%$ of the total emission. Since the shapes of the HI profiles are similar, the difference may be explained better by the gain drift and the uncertain flux calibration in the old single dish data. A significant amount of extended emission is missed but about $40 \%$ of the $\mathrm{DnC} \mathrm{HI}$ flux is recovered in the $\mathrm{CnB}$ data. 


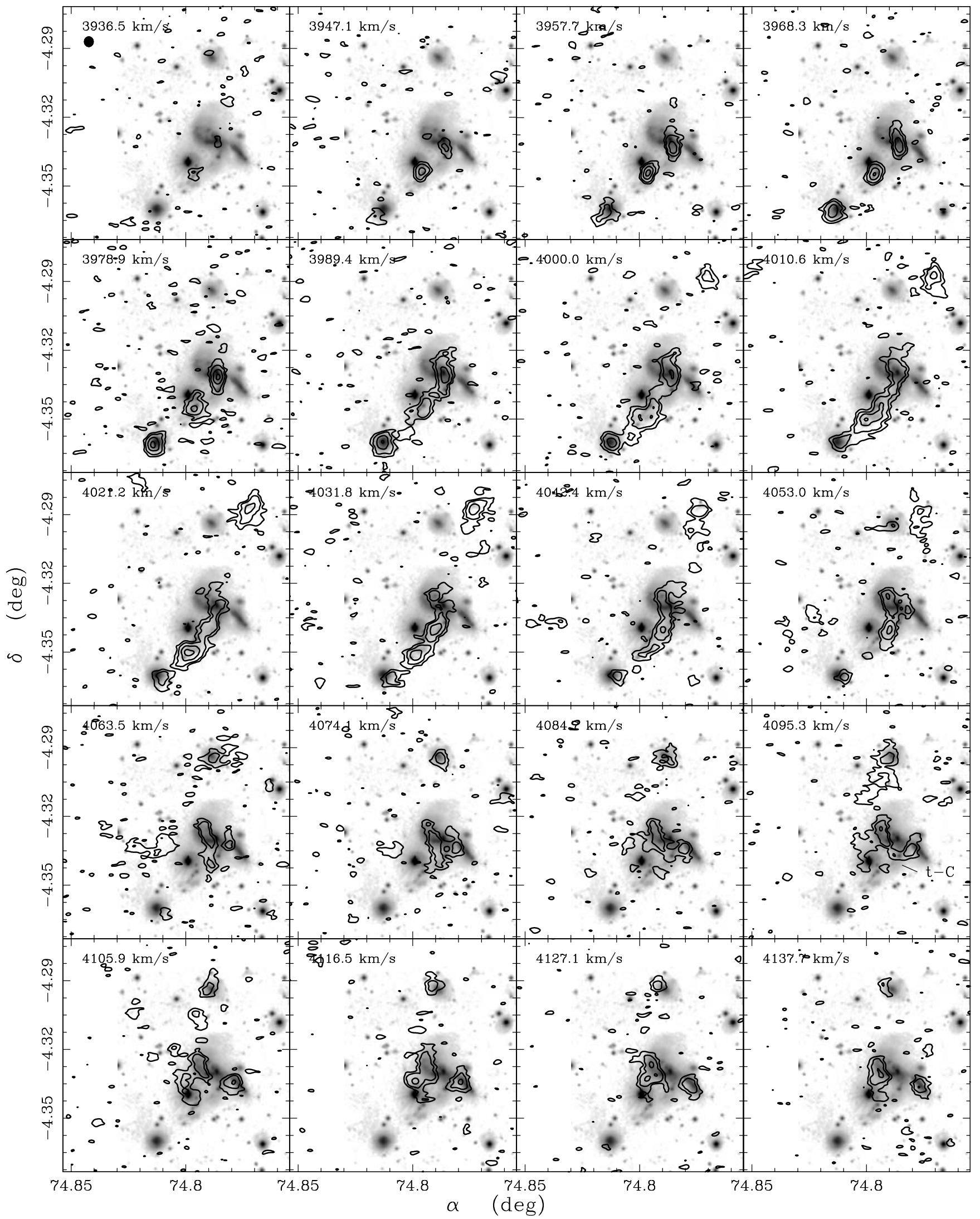

Fig. 4. Channel maps of the $21 \mathrm{~cm}$ line radiation obtained with the VLA CnB configuration, superimposed on the $B$-band image median smoothed by $4^{\prime \prime} \times 4^{\prime \prime}$. Heliocentric velocities are indicated in each panel. Contours correspond to $-3.0,3.0,6.1,11.8$ and $17.9 \mathrm{~K}$, and the rms noise of the maps is $1.2 \mathrm{~K}$. The synthesized beam $\left(15\right.$." $^{\prime} 8 \times 14$." $\left.^{\prime}\right)$ is plotted in the upper left panel. 


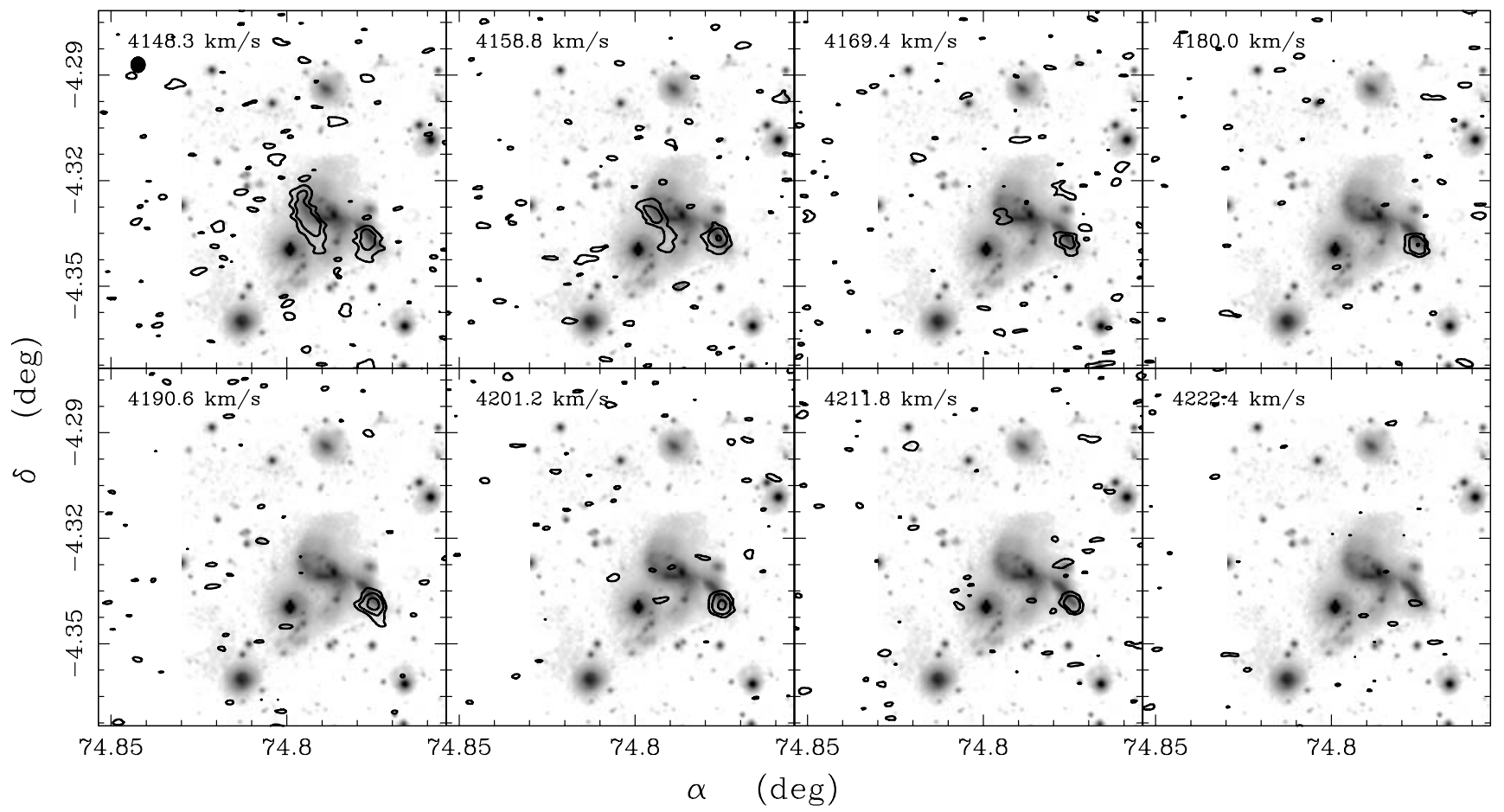

Fig. 4. continued.
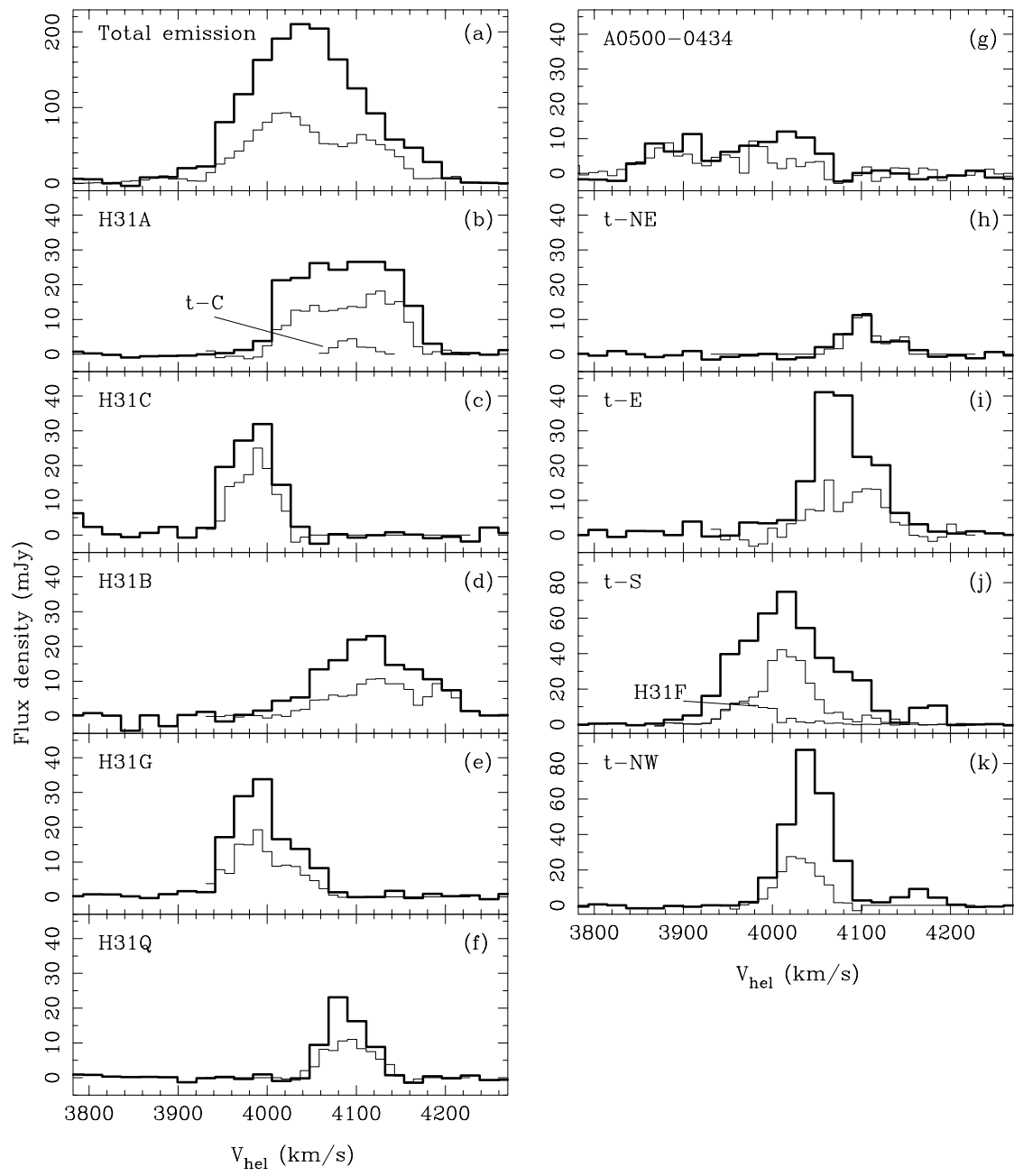

Fig. 5. HI line profiles for the individual HI features. Thick lines show the spectra derived from the DnC array data while the thin lines show the spectra from the $\mathrm{CnB}$ array data. Panel b) also displays the weak emission in the bridge joining H31A and H31B (named as t-C, see Sect. 4.1). The HI spectrum obtained at the location of $\mathrm{H} 31 \mathrm{~F}$ is shown in panel $\mathbf{j}$ ). 
Table 2. HI emission parameters.

\begin{tabular}{lcccccc}
\hline \hline Object & $\begin{array}{c}V^{a} \\
\left(\mathrm{~km} \mathrm{~s}^{-1}\right)\end{array}$ & $\begin{array}{c}\text { Velocity range } \\
\left(\mathrm{km} \mathrm{s}^{-1}\right)\end{array}$ & $\begin{array}{c}\Delta v_{20}^{b} \\
\left(\mathrm{~km} \mathrm{~s}^{-1}\right)\end{array}$ & $\begin{array}{c}\Delta v_{50}{ }^{c} \\
\left(\mathrm{~km} \mathrm{~s}^{-1}\right)\end{array}$ & $\begin{array}{c}T_{\max }{ }^{d} \\
(\mathrm{~K})\end{array}$ & $\log \left(M(\mathrm{HI}) / M_{\odot}\right)^{e}$ \\
\hline H31A & 4090 & $4021-4180$ & 169.2 & 169.2 & 13.6 & 9.30 \\
H31B & 4122 & $4042-4222$ & 190.6 & 126.3 & 13.6 & $9.28^{(g)}$ \\
t-C & 4095 & $4074-4095$ & 52.9 & 21.3 & 6.3 & 8.00 \\
H31C & 3984 & $3936-4021$ & 85.8 & 64.3 & 20.0 & 9.20 \\
H31G & 4005 & $3937-4064$ & 128.7 & 42.9 & 20.1 & 9.28 \\
H31Q & 4090 & $4053-4138$ & 84.9 & 42.4 & 9.8 & 8.95 \\
A0500-0434 & 3952 & $3852-4053$ & 233.1 & 233.1 & 9.5 & 9.04 \\
t-E & 4080 & $4042-4138$ & 107.5 & 63.2 & 6.8 & 9.41 \\
t-S & 4016 & $3936-4116$ & 190.8 & 126.8 & 20.5 & 9.81 \\
H31F & 3968 & $3937-4011$ & 74.6 & 63.8 & 19.1 & 8.78 \\
t-NE & 4111 & $4085-4117$ & 84.9 & 42.4 & 7.9 & 8.48 \\
t-NW & 4048 & $4000-4064$ & 85.0 & 63.3 & 18.3 & 9.59 \\
\hline Total DnC & 4059 & & 232.7 & 148.3 & 20.5 & 10.37 \\
Total CnB & 4053 & $3937-4222$ & 222.3 & 158.6 & 20.5 & 9.99 \\
Total single dish ${ }^{f}$ & 4041 & & 212 & 147 & 0.2 & 10.20 \\
\hline
\end{tabular}

${ }^{a}$ Central velocity at $20 \%$ of the peak emission in the integrated spectra obtained from the DnC array data, except for $\mathrm{H} 31 \mathrm{~F}$ and t-C, only resolved in the $\mathrm{CnB}$ array.

${ }^{b}$ Line width measured at $20 \%$ of the peak emission in the same data indicated in ${ }^{a}$.

${ }^{c}$ Line width measured at $50 \%$ of the peak emission in the same data indicated in ${ }^{a}$.

${ }^{d}$ Peak temperature measured in the same data indicated in ${ }^{a}$.

e $M(\mathrm{HI})=2.36 \times 10^{5} \times D(\mathrm{Mpc})^{2} \times S_{\mathrm{HI}} \Delta V\left(\mathrm{Jy} \mathrm{km} \mathrm{s}{ }^{-1}\right)$, where $D=54.3 \mathrm{Mpc}$ and $S_{\mathrm{HI}} \Delta V$ is the integrated emission in the same data indicated in ${ }^{a}$.

$f$ Obtained from Williams \& Rood (1987).

$g$ The calculated mass includes the emission from $\mathrm{t}-\mathrm{C}$ unresolved with the DnC-array data. The t-C mass is given below based on the $\mathrm{CnB}$ data.
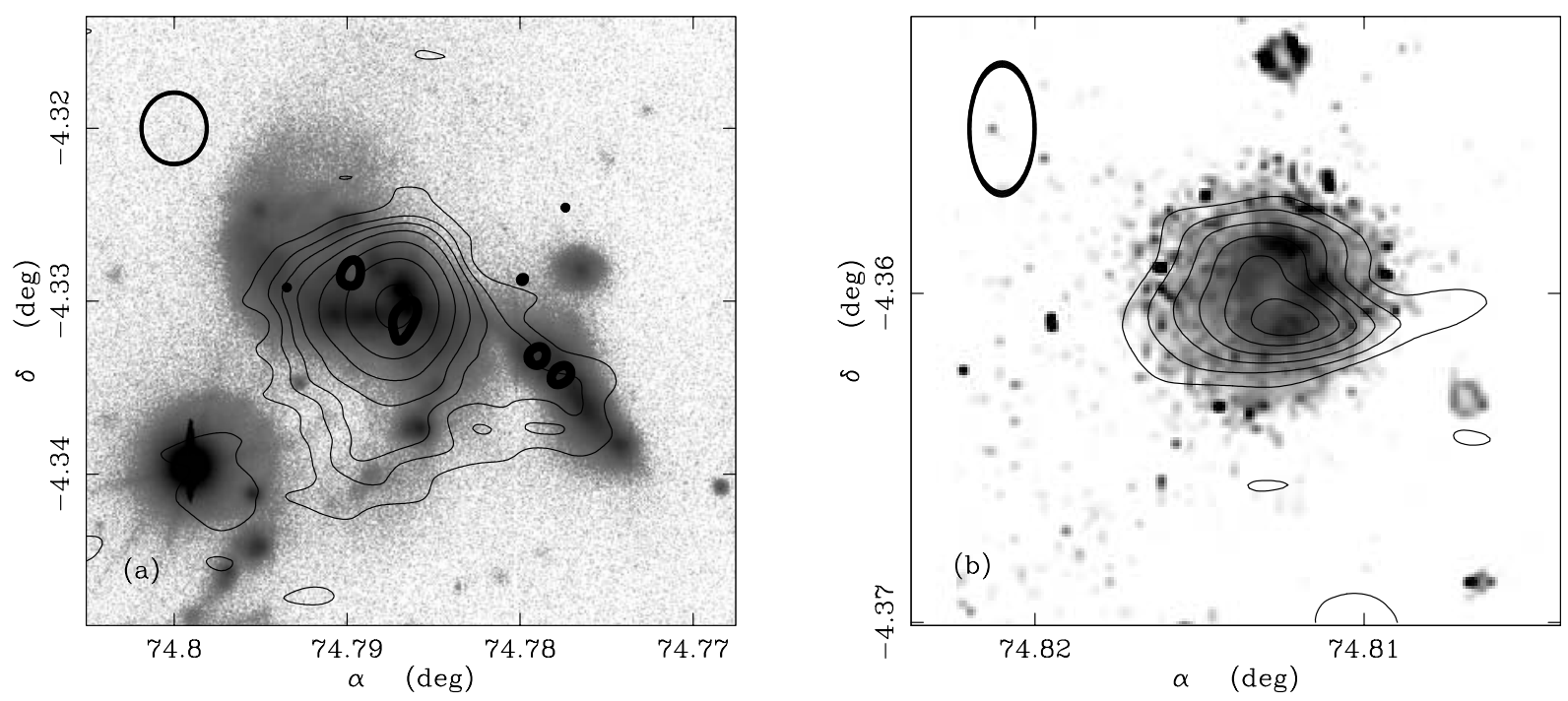

Fig. 6. a) $1.4 \mathrm{GHz}$ ratio continuum emission associated to the central area of HCG 31 derived from the line-free channels of the CnB array data. The contours correspond to 1.1, 2.1, 3.2, 4.8, 8.0, 14.3 and $27.1 \mathrm{~K}$ (rms noise $0.37 \mathrm{~K}$ ) and are superposed on a $B$ image of the group. Shown as thick contours is the CO emission from Yun et al. (1997). The synthesized beam $\left(155^{\prime \prime} 7 \times 14\right.$.' $\left.^{\prime}\right)$ is plotted in the upper left. b) $1.4 \mathrm{GHz}$ continuum emission image of $\mathrm{H} 31 \mathrm{G}$ is shown superposed on a $B-I$ color image. Radio continuum closely follows the redder (lighter) $B-I$ color regions. The contours correspond to $1.6,2.6,3.7,4.8,5.8,6.9$ and $7.9 \mathrm{~K}(1 \sigma=0.85 \mathrm{~K})$. The synthesized beam $\left(15^{\prime \prime} 1 \times 7{ }^{\prime \prime} 5\right)$ is plotted in the upper left.

\section{3. $1.4 \mathrm{GHz}$ continuum}

The $1.4 \mathrm{GHz}$ radio continuum distribution has been obtained from the line free channels in both data sets, and the resulting continuum map, Fig. 6a, is a significant improvement over the continuum image presented by W91. The new continuum map is produced using natural weighting of the uv data in order to maximize the sensitivity, and a synthesized beam of

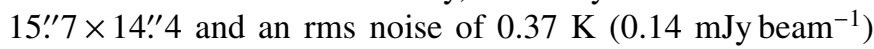
are achieved. The peak of the continuum emission is 
located at the intersection of galaxies $\mathrm{H} 31 \mathrm{~A}$ and $\mathrm{H} 31 \mathrm{C}$, unlike the continuum image shown by W91, who identified H31C as the radio continuum source. The radio continuum peak is also shifted with respect to the $\mathrm{CO}$ emission, plotted as thick contours reproduced from Yun et al. (1997). An extension in the direction of $\mathrm{H} 31 \mathrm{~F}$ and $\mathrm{G}$ is also evident. A new continuum source is detected at the position of H31B at a level of $1.1 \pm 0.2 \mathrm{mJy}$, nearly coincident with the optical center. The radio continuum image for $\mathrm{H} 31 \mathrm{G}$ has been produced using robust weighting of the uv data in order to to better resolve the continuum structure(synthesized beam of 15 !' $^{\prime} \times 7$.' $^{\prime}$ ). It was marginally detected by W91, and is clearly detected now (Fig. 6b), peaking on the opposite side of the bluest region in this galaxy. The total detected $1.4 \mathrm{GHz}$ continuum fluxes for $\mathrm{H} 31 \mathrm{AC}, \mathrm{H} 31 \mathrm{~B}$ and $\mathrm{H} 31 \mathrm{G}$ are $22 \pm 3$, $2.1 \pm 0.3$, and $3.3 \pm 0.5 \mathrm{mJy}$, respectively. While the $1.4 \mathrm{GHz}$ flux for $\mathrm{H} 31 \mathrm{AC}$ as well as $\mathrm{H} 31 \mathrm{G}$ are within the observed scatter in the well known FIR-radio correlation (0.26 in dex; Yun et al. 2001), H31b shows some excess radio continuum emission. Galaxies hosting radio-quiet AGNs typically show radioexcess of a factor 2-4 (see Yun \& Carilli 2002), and the radio excess seen in $\mathrm{H} 31 \mathrm{~b}$ is consistent with the presence of a radio-quiet AGN.

\section{The members and environment of HCG 31}

The three brightest members of HCG $31(\mathrm{H} 31 \mathrm{~A}, \mathrm{H} 31 \mathrm{~B}$ and $\mathrm{H} 31 \mathrm{C})$ are located within a diameter of only $14 \mathrm{kpc}\left(0^{\prime} \cdot 9\right)$. Adding H31G increases the group diameter to $32 \mathrm{kpc}$ (R90), and $\mathrm{H} 31 \mathrm{Q}$ expands the group diameter further to $66 \mathrm{kpc}$. We determine a mean heliocentric velocity for the group of $4076 \mathrm{~km} \mathrm{~s}^{-1}$, a velocity dispersion of $60 \mathrm{~km} \mathrm{~s}^{-1}$ and a mean separation between galaxies of $10 \mathrm{kpc}$, based on the optical velocities of H31A, H31B, H31C (Hickson 1993), H31G (R90) and H31Q (Richer et al. 2002).

We have searched for neighbors of HCG 31 in a 1 Mpc radius $\left(1^{\circ}\right)$ in the CfA catalog (Huchra et al. 1993) and NED see Fig. 7. The inner $0.5 \mathrm{Mpc}$ contains 1 galaxy with redshift and magnitude similar to the HCG 31 members whose discovery is reported in this paper (Sect. 4.5, A0500-0434). One other galaxy with no previously known redshift is found $28^{\prime}$ (442 kpc) to the north (NPM1G -04.0218), but our own NOT spectrum shows it to be a background galaxy $(z=0.087)$. Between the projected distance of 0.5 and $1 \mathrm{Mpc}$, NGC 1729 is at the same redshift as the group $\left(v_{r}=3644 \mathrm{~km} \mathrm{~s}^{-1}\right.$, $m_{B}=13,0.91 \mathrm{Mpc}$ to the NW), and thirty additional galaxies are known, including the three known to be in the background. The 18 faint galaxies with no known redshifts cluster around Abell cluster $531(z=0.94)$, and they are likely the cluster members. Therefore A0500-0434 (see Sect. 4.5) is probable the only galaxy dynamically associated to the group, located $239 \mathrm{kpc}$ south of the group center and similar in redshift and size to each member of HCG 31. From these considerations, we conclude that HCG 31 is located in a low density region.

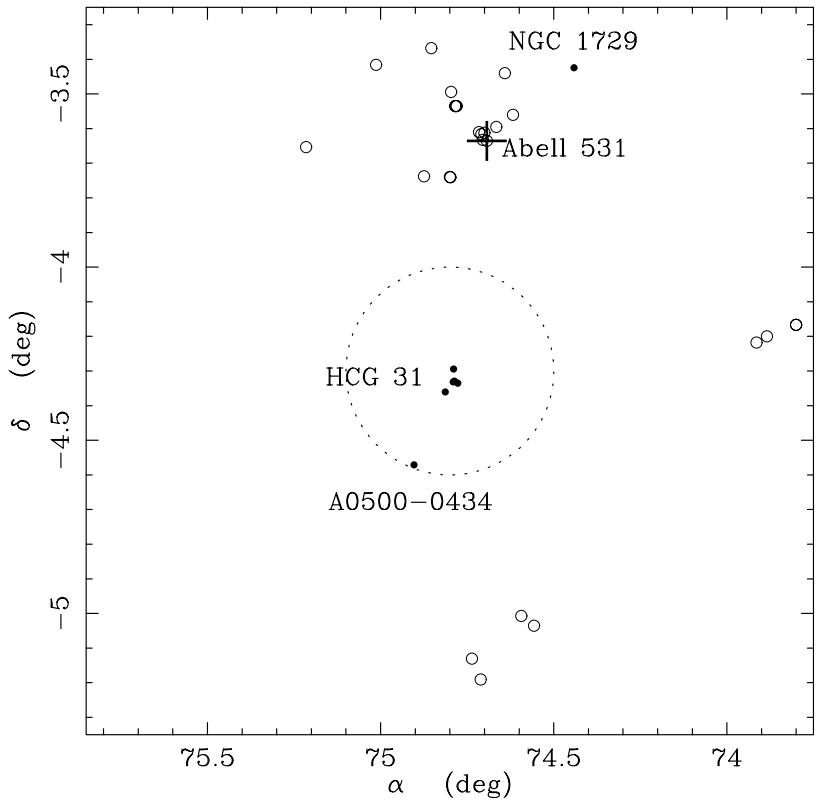

Fig. 7. A diagram of the environment of HCG 31. Filled circles identify the members of the group and galaxies with similar redshift. The galaxies without redshift data are represented by open circles, and the center of Abell 531 is marked with a cross. The dotted circle indicates the VLA primary beam. Galaxies with a clearly different redshift from the group are omitted.

\section{The galaxies}

\section{1. $H 31 A C$}

H31 A and H31C have a disrupted appearance not only in the optical light, but also in HI, with at least three velocity components overlapping. Hence the intensity weighted mean velocity of the HI emission is confusing and possibly even misleading. Nevertheless, we were able to separate the HI gas associated with the individual galaxies $\mathrm{H} 31 \mathrm{~A}$ and $\mathrm{C}$ using the $\mathrm{HI}$ morphology and kinematics as described below.

H31A.- The brightest knots of this galaxy align in the E-W direction, with fainter emission extending to the north (a $B$-band image is shown in Fig. 8a). These bright knots are prominent in the sharpened $R$-band image (Fig. 2d) constructed by subtracting a $3^{\prime \prime} \times 3^{\prime \prime}$ median filtered image. Two of these knots are embedded in faint emission orientated with PA $\sim 88^{\circ}$, the brightest one with a round shape characteristic of a small bulge. The $B-I$ color image (Fig. 2c), shows a distinct red feature $(B-I=1.4)$ with $\mathrm{PA}=65^{\circ}$. Its colors are much redder than those of $\mathrm{H} 31 \mathrm{C}$ (see below) and suggest that it is tracing the disk of H31A.

Approximately $3 \times 10^{9} M_{\odot}$ of atomic gas are associated with the main body of H31A, generally correlating well with the optical features. The channel maps peak on the two brightest optical knots (Fig. 4). An arc-like bridge (t-C) joins H31A and $B\left(V=4074-4095 \mathrm{~km} \mathrm{~s}^{-1}\right)$, while the two tidal features tE and t-NE appear to connect with H31A. We show in Fig. 5b the global HI profiles obtained from the $\mathrm{DnC}$ and $\mathrm{CnB}$ array data, including the weak emission corresponding to $\mathrm{t}-\mathrm{C} \mathrm{ob}-$ tained from the $\mathrm{CnB}$ array data. The corresponding parameters are given in Table 2. 


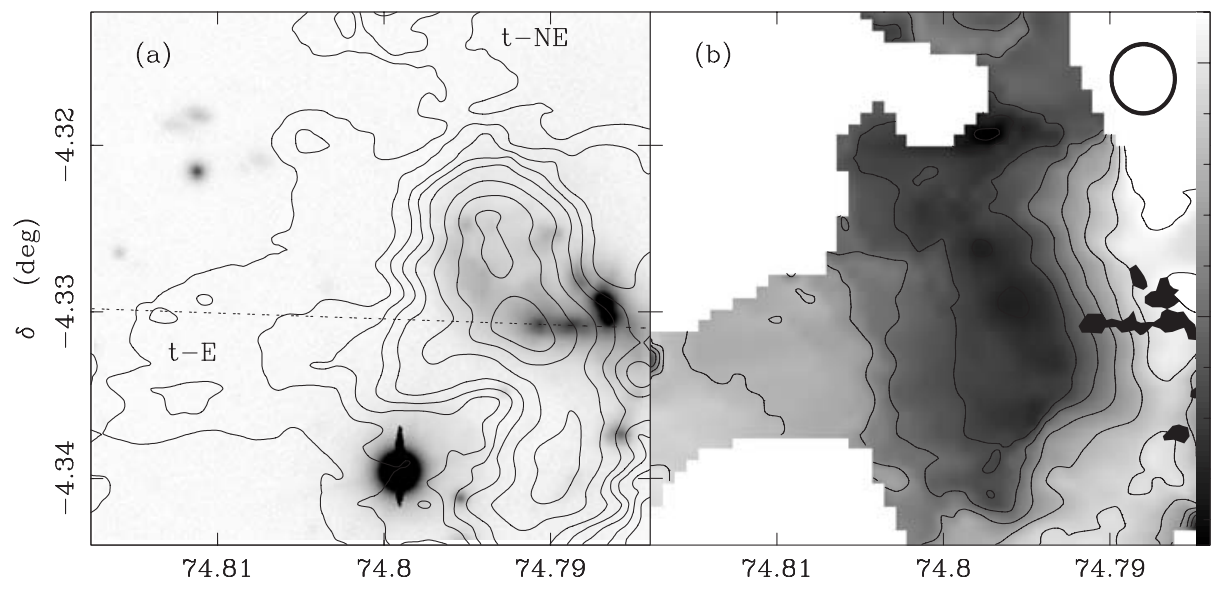

4050

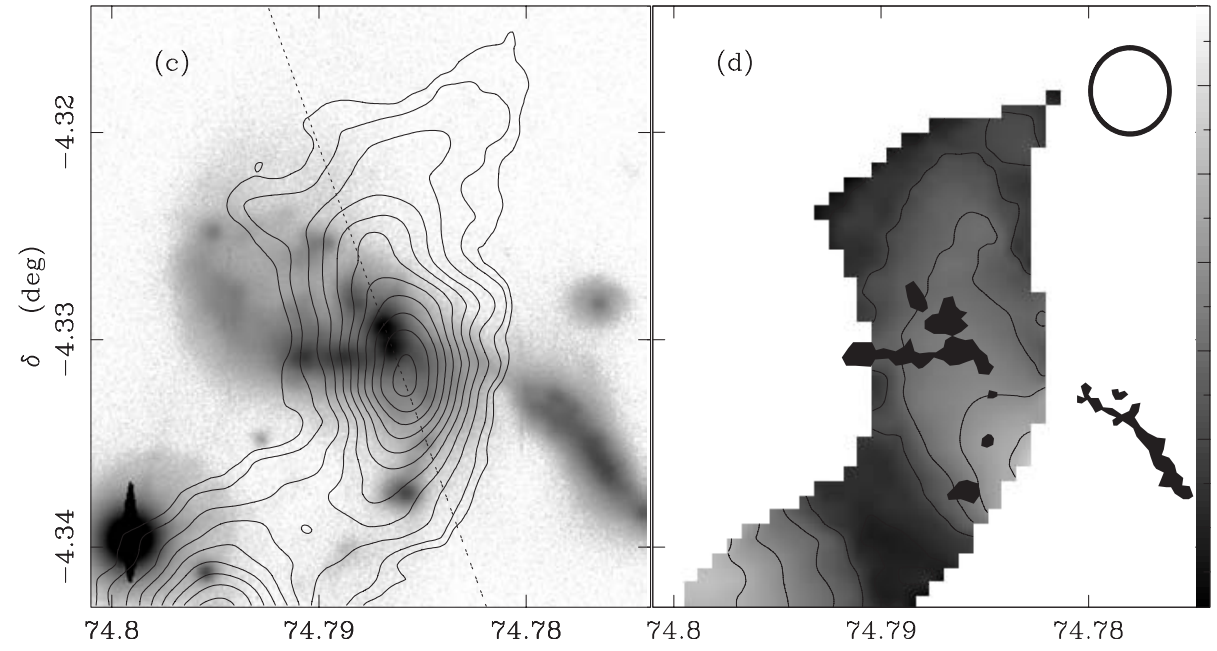

3960

3970

3980

3990

4000

4010

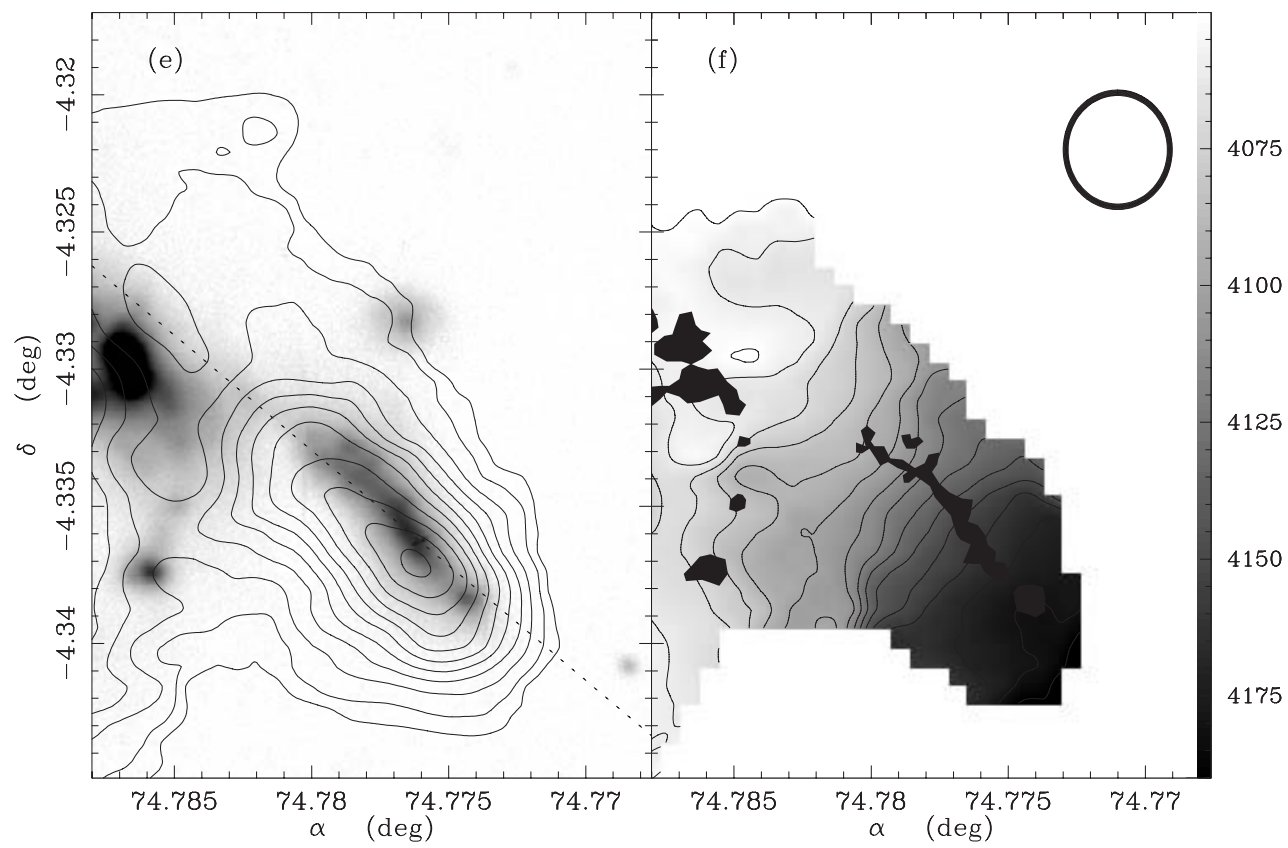

Fig. 8. a), c) and e) present respectively the HI column density (CnB-array data) of H31A $\left(V=4021-4180 \mathrm{~km} \mathrm{~s} \mathrm{~s}^{-1}\right), \mathrm{H} 31 \mathrm{C}(V=$ $\left.3937-4021 \mathrm{~km} \mathrm{~s}^{-1}\right)$ and H31B $\left(V=4042-4222 \mathrm{~km} \mathrm{~s}^{-1}\right)$ overplotted on a $B$-band image in a logarithmic gray scale. The contours are 1.0, 2.4, 3.8, 5.2, 6.9, 9.6, 13.0, 16.5, 19.9 and $23.3 \times 10^{20}$ atoms $\mathrm{cm}^{-2}$ for $\mathrm{H} 31 \mathrm{~A}$, and $0.9,1.9,3.8,5.7,7.5,9.4,11.3,13.2,15.1,17.0$, and $18.8 \times 10^{20}$ atoms $\mathrm{cm}^{-2}$ for $\mathrm{H} 31 \mathrm{C}$ and $\mathrm{B}$. The dashed lines indicate the PA of $88^{\circ}, 20^{\circ}$ and $40^{\circ}$ used for the position velocity cut plotted in Figs. 9a-c for H31A, H31C and H31B respectively. b), d) and f) show the first-order moment of the radial velocity field of H31A, H31C and H31B where both iso-velocity contours (H31A: 3950 to $4020 \mathrm{~km} \mathrm{~s}^{-1}$, step $15 \mathrm{~km} \mathrm{~s}^{-1}$; H31C: 3950 to $4020 \mathrm{~km} \mathrm{~s}^{-1}$, step of $7 \mathrm{~km} \mathrm{~s}$; H31B: 4050 to $4190 \mathrm{~km} \mathrm{~s}^{-1}$; step $10 \mathrm{~km} \mathrm{~s}^{-1}$ ) and gray-scale are shown, together with a sketch of the sharpened $\mathrm{R}$ image of Fig. $2 \mathrm{~d}$. The scale goes as in the wedge where the numbers indicate heliocentric velocities in $\mathrm{km} \mathrm{s}^{-1}$. The beam size is 15 ." $^{\prime} \times 14$." $^{\prime}$ and is plotted in the upper right. 

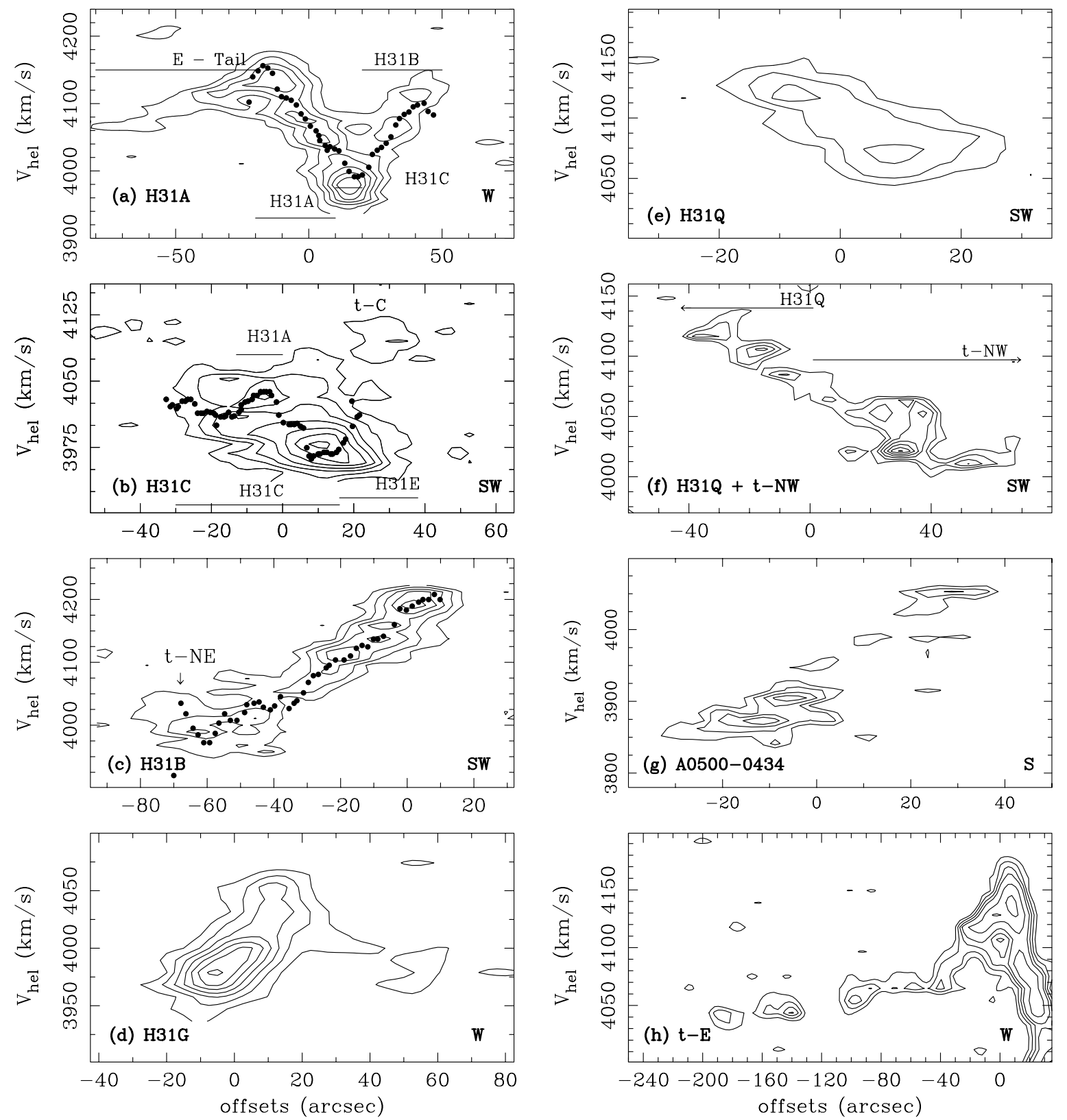

Fig. 9. Position-velocity diagrams of major HI features obtained along the position angles of a) $88^{\circ}$, b) $20^{\circ}$, c) $40^{\circ}$, d) $90^{\circ}$, e) $50^{\circ}$, f) $70^{\circ}$, g) $22^{\circ}$ and h) $97^{\circ}$. The plotted levels are 2.6, 5.2, 7.8, 10.5, 13.1, 15.7 and $18.3 \mathrm{~K}$, except for $\mathbf{f}$ ) where we plot 2.6, 3.3, 3.9, 4.6, 5.2, 5.9 and 6.5 K, g) with 2.6, 3.9, 5.2 and $6.5 \mathrm{~K}$ and $\mathbf{h}$ ) with $2.6,3.9,5.2,6.5,9.1,11.8$ and $14.4 \mathrm{~K}$. The offsets are given with respect to the peak position in our optical image, except for $\mathbf{h}$ ) which does not show optical counterpart, and has been arbitrarily chosen as the position where this feature separates from the disk of H31A. The dots correspond to the optical rotation curve obtained by R90, shifted by the amount given in the text. The synthesized beam is 15 ". $8 \times 14$." 5 .

The velocity range $4021-4180 \mathrm{~km} \mathrm{~s}^{-1}$ contains the bulk of H31A emission while largely excluding emission from H31C (see Fig. 8a). Ignoring the emission associated with t-E, the HI kinematics of H31 A (Fig. 8b) shows a velocity gradient of $\sim 140 \mathrm{~km} \mathrm{~s}^{-1}$ with a PA of $109^{\circ}$. The central velocity is approximately $4060 \mathrm{~km} \mathrm{~s}^{-1}$, and the kinematical center position is $\alpha(1950)=04^{\mathrm{h}} 59^{\mathrm{m}} 09^{\circ} .4, \delta(1950)=-04^{\circ} 19^{\prime} 53^{\prime \prime}, \sim 5^{\prime \prime}$ east of the brightest optical knot. At larger radii the position angle of the kinematical major axis twists by $-58^{\circ}$, suggesting a warping of the gas. The optical velocities obtained by R90 along PA $=88^{\circ}$ (which are consistent with those obtained by
Richer et al. 2003) with the HI position-velocity diagram along the same direction are compared in Fig. 9a. A good agreement is achieved if the slit is shifted by $5^{\prime \prime}$ to the west. The rotation curve of H31A was described by R90 as irregular due to the velocity inversion that occurs at $\sim 20^{\prime \prime}$. Our HI data show that it is in fact due to overlapping of $\mathrm{H} 31 \mathrm{~A}$ with three different components: t-E for $\leq-20^{\prime \prime}, \mathrm{H} 31 \mathrm{C}$ at $\sim 10^{\prime \prime}$, and H31B for $r>20^{\prime \prime}$ (see channel maps in Fig. 4).

H31C.- This is a luminous Wolf Rayet galaxy (Conti 1991) with a $\mathrm{N}-\mathrm{S}$ orientation as suggested by its morphology and kinematics. The two brightest optical knots are aligned along 

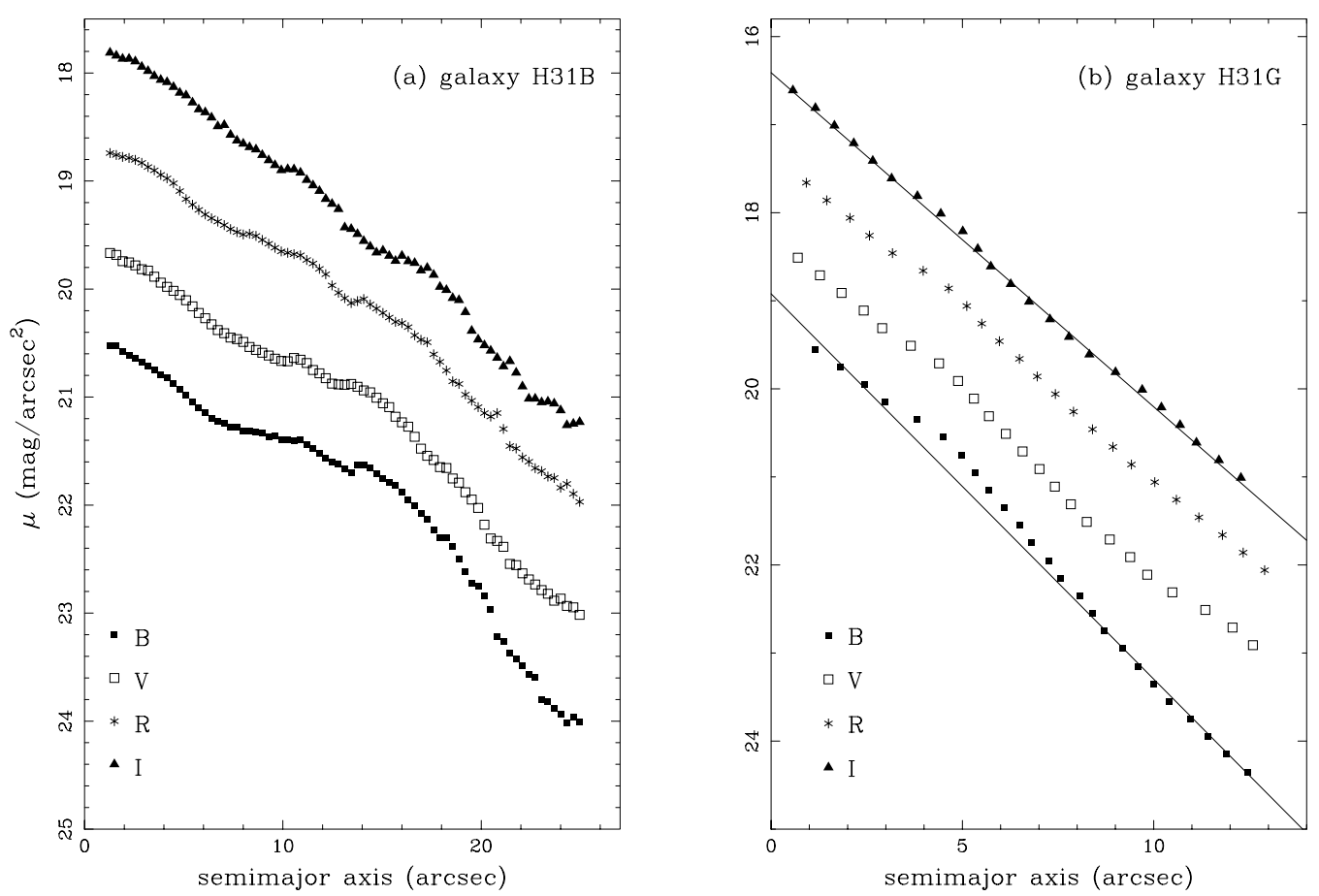

Fig. 10. Surface brightness profiles of a) $\mathrm{H} 31 \mathrm{~B}$ and b) $\mathrm{H} 31 \mathrm{G}$ as a function of the semi-major axis distance. They have been corrected for galactic absorption by 0.25 mag in $\mathrm{B}, 0.19$ mag in $V, 0.14$ mag in $R$, and 0.09 mag in $I$. The $V, R$ and $I$ profiles have been shifted upwards respectively by $0.5,1$ and 1.5 mag for clarity.

a PA of $12.5^{\circ}$ (see Fig. 8c) and correspond to actively starforming regions (Iglesias-Páramo \& Vílchez 1997; Johnson et al. 1999). The intense blue colors of $\mathrm{H} 31 \mathrm{C}$ ( $B-I$ color ranges from 0.5 and 0.7 ) show up north and south of the red optical component associated to $\mathrm{H} 31 \mathrm{~A}$ (Fig. 2c), suggesting that $\mathrm{H} 31 \mathrm{C}$ lies behind $\mathrm{H} 31 \mathrm{~A}$ along the line-of-sight.

The $\mathrm{HI}$ emission in $\mathrm{H} 31 \mathrm{C}$ is detected in the velocity range $3937-4021 \mathrm{~km} \mathrm{~s}^{-1}$. The HI spectrum is shown in Fig. 5c. The HI is elongated in all channel maps with PAs ranging between $0^{\circ}$ and $20^{\circ}$ (see Fig. 4). The integrated atomic emission shows the same orientation as the optical source (see Fig. 8c) except for a weak HI extension to the NW that belongs to the blueshifted component of t-NW and has no obvious optical counterpart. The emission peak with a velocity of $V=3979 \mathrm{~km} \mathrm{~s}^{-1}$ is located $8^{\prime \prime}$ to the south of the bright optical knots. This shift could be explained if the real center of $\mathrm{H} 31 \mathrm{C}$ is hidden behind $\mathrm{H} 31 \mathrm{~A}$ or if its $\mathrm{HI}$ disk is severely disrupted. A velocity gradient of $\sim 60 \mathrm{~km} \mathrm{~s}^{-1}$ with an approximate PA of $30^{\circ}$ is derived, bending to the $\mathrm{NW}$ in coincidence with the above indicated extension (see Fig. 8d). The optical rotation curve obtained by $\mathrm{R} 90$ along $\mathrm{PA}=20^{\circ}$ (similar to the one obtained by Richer et al. 2003 along the same direction) is compared in Fig. 9b with the corresponding HI velocities. A shift of $10^{\prime \prime}$ to the NE had to be made to the optical curve in order to optimize the match. As the channel maps suggest, the bump found in the optical curve of H31C from $r=-13^{\prime \prime}$ to $0^{\prime \prime}$ is probably produced by the overlapping emission from H31A. Emission from $\mathrm{H} 31 \mathrm{E}$ and $\mathrm{t}-\mathrm{C}$ are also identified in the same position-velocity diagram.

In summary, we were able to distinguish and separate the $\mathrm{HI}$ emission associated with the disks of H31A and H31C using the morphology and kinematics in the new high resolution VLA HI data and new optical images. The kinematics of the two galaxies show almost perpendicular velocity gradients identifying two separated galaxies. The optical images point in this direction, indicating the presence of a disk in H31 A with a clearly different $B-I$ color index from $\mathrm{H} 31 \mathrm{C}$.

\section{2. $H 31 B$}

$\mathrm{H} 31 \mathrm{~B}$ is a lopsided nearly edge-on $\left(i \sim 77^{\circ}\right)$ galaxy whose optical light extends $20 \%$ more to the NE, where it appears to bifurcate. The presence of $\mathrm{CO}$ emission at the break point suggests that it is not real but a result of dust obscuration. Similar attenuation of the optical light is found at inner radii coinciding with a second CO clump. The disk of H31B overlaps with H31AC at its NE edge (Fig. 1; also Iglesias-Páramo \& Vílchez 1997). The $V$ band isophotal contours twist from a PA $=35^{\circ}\left(r<7^{\prime \prime}\right)$ to $55^{\circ}$ ( $r>7^{\prime \prime}$, Fig. 2a). A similar twist in the HI component could not be resolved with the angular resolution of the present data. The $B-I$ color image shows a red area tracing the disk of H31B, plus blue knots in the outer parts (see Fig. 2c).

The surface brightness (SB) profiles in the four filters as a function of the semimajor axis are shown in Fig. 10a. As can be seen in the figure, type-II SB profiles, more pronounced in the blue, are present in all the bands, having a portion of their inner brightness profiles lying below the inward disk extrapolation (Freeman 1970). This prevents us from obtaining a realistic bulge/disk decomposition. The light profiles have steeper surface brightness distribution in the outer regions, characteristic of type-II SB profile systems (Anderson et al. 2004). We discuss it in further detail in Sect. 6. 


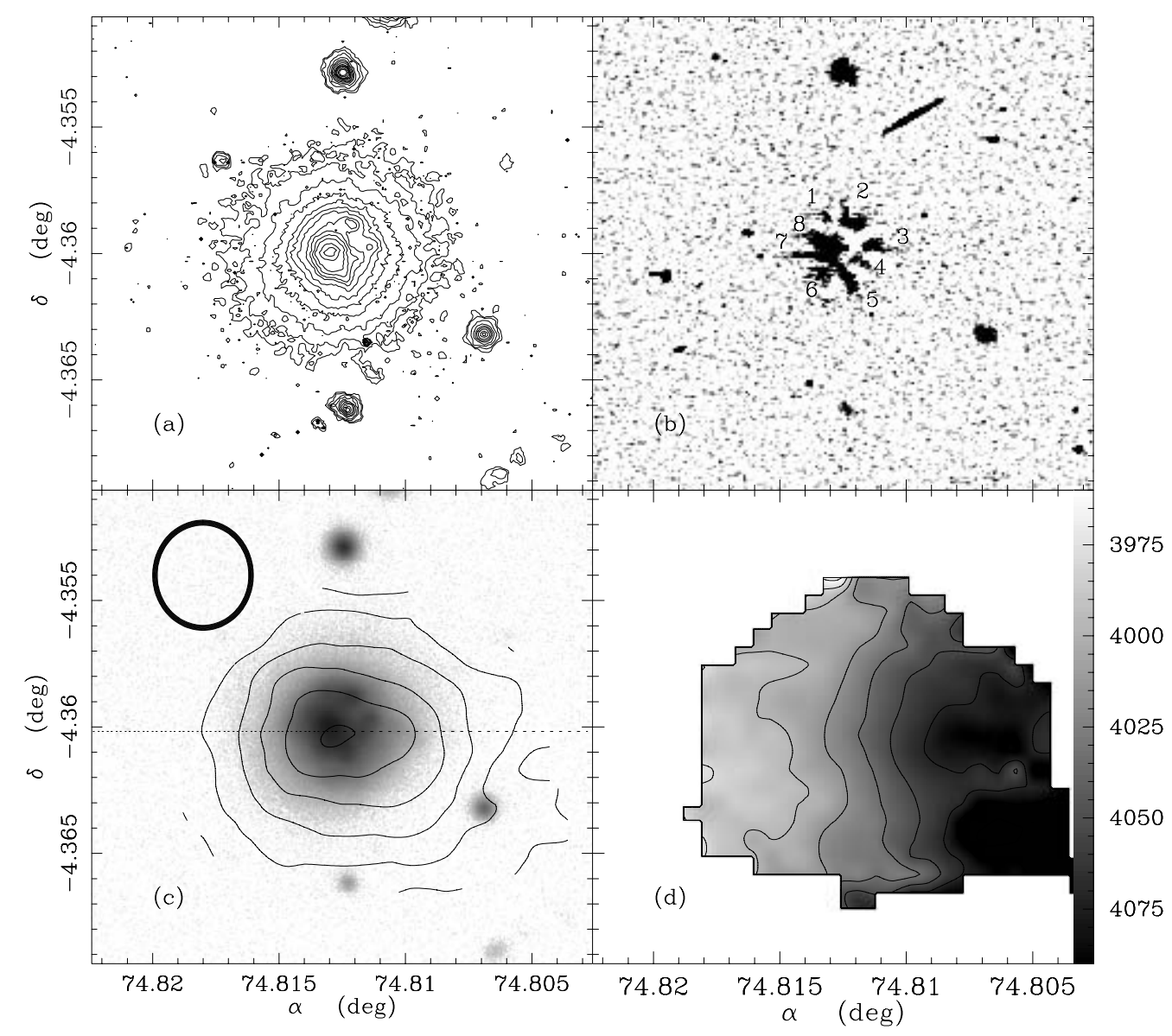

Fig. 11. a) A contour plot of the $B$-band image of H31G. Contour values are 19.6, 19.8, 20.0, 20.2, 20.4, 20.6, 20.8, 21.2, 21.8, 22.6, 23.4 and $24.2 \mathrm{mag} \mathrm{arcsec}^{-2}$. b) A sharpened image obtained by subtracting of a $3^{\prime \prime} \times 3^{\prime \prime}$ median filtered image. The parameters of the labeled knots are given in Table 3. c) The CnB-array HI image of H31G integrated over the velocity range of $3937-4064 \mathrm{~km} \mathrm{~s}^{-1}$. The HI column density contours are $0.9,3.8,7.5,11.3,15.1$, and $18.8 \times 10^{20}$ atoms $\mathrm{cm}^{-2}$. A $B$-band image of $\mathrm{H} 31 \mathrm{G}$ shown in a logarithmic gray scale representation. The dotted line indicates the PA of $90^{\circ}$ used for the position velocity plot shown in Fig. 9d. The synthesized beam $\left(15^{\prime \prime} 8 \times 14\right.$.' $\left.^{\prime}\right)$ is plotted in the upper left. d) First moment of the radial velocity image of H31G. Both iso-velocity contours and gray-scale are shown. The contours range between 3930 and $4060 \mathrm{~km} \mathrm{~s}^{-1}$ in steps of $10 \mathrm{~km} \mathrm{~s}^{-1}$.

The $\mathrm{HI}$ emission of $\mathrm{H} 31 \mathrm{~B}$ is detected in the velocity range $4042-4222 \mathrm{~km} \mathrm{~s}^{-1}$ and correlates generally well with the optical, except for its SW half where an offset of $5^{\prime \prime}$ to the south is found (Fig. 8e). It extends further than the optical emission by $25 \%$ to the SW and by $35 \%$ to the NE, measured at a column density of $3 \times 10^{20} \mathrm{~cm}^{-2}$. This asymmetry is reflected in the $\mathrm{HI}$ integrated spectrum shown in Fig. 5d. The HI velocity field (Fig. 8f) is consistent with a rotating disk with a velocity gradient of $140 \mathrm{~km} \mathrm{~s}^{-1}$ and a PA of $\sim 50^{\circ}$. The most intense and regular emission is located in the range $4090-4222 \mathrm{~km} \mathrm{~s}^{-1}$. The symmetry center is located at $4150 \mathrm{~km} \mathrm{~s}^{-1}$ and $7 "$ to the NE of the optical peak. The HI kinematics is in good agreement with the $\mathrm{H} \alpha$ rotation curve obtained by $\mathrm{R} 90$ along a PA of $40^{\circ}$ (Fig. 9c) and by Richer et al. (2003). Both HI and optical velocities invert their trends at $60^{\prime \prime}$ to the NE, coincident with the tidal feature t-NE (Sect. 5.1).

\section{3. $H 31 G$}

$\mathrm{H} 31 \mathrm{G}$ has been classified as an irregular and peculiar Markarian galaxy (Mk 1090) with giant HII regions. It is located 2.4 $(36 \mathrm{kpc})$ south of the group center, and its line of sight velocity differs from the mean group velocity by only $115 \mathrm{~km} \mathrm{~s}^{-1}$. It has a moderately high IR luminosity $\left(L_{\mathrm{FIR}}=\right.$ $2 \times 10^{9} L_{\odot}$, Yun et al. 1997), but no CO emission is detected with a $\mathrm{H}_{2}$ mass limit of $4.1 \times 10^{8} M_{\odot}$ (Verdes-Montenegro et al. 1998).

The $R$-band image of H31G (Fig. 11a) shows several bright knots that appear smoother in the $I$-band image. The axial ratio of the outer isophotes $(0.95$ at $r \sim 7 \mathrm{kpc})$ corresponds to a very small inclination $\left(\sim 18^{\circ}\right)$. We have identified 8 knots in the sharpened image produced by subtracting a $3^{\prime \prime} \times 3^{\prime \prime}$ median filtered image (Fig. 11b). Those knots are detected in $\mathrm{H} \alpha$ by Johnson \& Conti (2000) and form an asymmetric ring of HII regions, probably as a result of less intense star forming activity or due to internal extinction. We favor the second interpretation since the radio continuum emission is quite homogeneus with respect to the $\mathrm{H} \alpha$ distribution in $\mathrm{H} 31 \mathrm{G}$, even considering the lower resolution of the radio data (Fig. 6). Table 3 lists the magnitudes and color indices of each optical knots and of the nucleus measured with an aperture of 2.' 9 in diameter. The total $B$-band magnitude and color index of the whole galaxy has been measured in an aperture of $25^{\prime \prime}$ in diameter. 
Table 3. Color indices for $\mathrm{H} 31 \mathrm{G}$ optical knots.

\begin{tabular}{llllll}
\hline \hline Knot & Aperture & $B_{\mathrm{mag}}$ & $B-V$ & $B-R$ & $B-I$ \\
\hline Galaxy & $25^{\prime \prime}$ & 14.87 & 0.43 & 0.90 & 1.23 \\
1 & 2.9 & 18.96 & 0.31 & 0.77 & 0.94 \\
2 & $2 . \prime 9$ & 18.56 & 0.26 & 0.70 & 0.90 \\
3 & 2.9 & 18.88 & 0.37 & 0.75 & 0.89 \\
4 & 2.9 & 18.54 & 0.31 & 0.77 & 1.05 \\
5 & 2.9 & 18.62 & 0.35 & 0.74 & 0.98 \\
6 & $2 . \prime 9$ & 18.50 & 0.40 & 0.80 & 1.16 \\
7 & $2 . ' 9$ & 19.21 & 0.55 & 0.93 & 1.29 \\
8 & 2.9 & 17.71 & 0.39 & 0.82 & 1.23 \\
\hline
\end{tabular}

The radial brightness profiles of $\mathrm{H} 31 \mathrm{G}$ in the four observed bands are shown in Fig. 10b as a function of the semimajor axis. The above indicated knots show up as bumps in the profiles at radii $4^{\prime \prime}$ to 7 ", increasingly pronounced to the bluer bands, supporting an HII nature. The exponential radial surface brightness profiles, obtained excluding the bumps from the fit, can be described by a disk profile $\left.\mu_{(} r\right)=\mu_{(0 D)}+a \times r\left(^{\prime \prime}\right)$, with $\mu_{(0 D)}=18.92,18.67,18.29$ and $17.87 \pm 0.05 \mathrm{mag} /\left({ }^{\prime \prime}\right)^{2}$ for $B, V, R$, and $I$ bands respectively. The corresponding values for $a$ are $0.43,0.40,0.38$ and $0.38 \pm 0.01$. The length scale of the disk $r_{0 D}$ is $\sim 0.7 \mathrm{kpc}$. In all cases the correlation coefficient is larger than 0.99 . The radial surface brightness analysis presented here clearly indicates that $\mathrm{H} 31 \mathrm{G}$ is a late-type galaxy with a very small bulge.

Although $\mathrm{H} 31 \mathrm{G}$ is located in the $\mathrm{t}-\mathrm{S}$ tail, its $\mathrm{HI}$ emission $\left(1.9 \times 10^{9} M_{\odot}\right.$; Fig. 5 e $)$ could be separated reasonably well from the t-S tail in our high resolution channel maps. The integrated emission map is obtained after masking off the t-S tail feature (see Fig. 11c, $V=3937-4064 \mathrm{~km} \mathrm{~s}^{-1}$ ). The atomic gas is well centered on the optical peak, and the mean velocity increases from west to east (see Fig. 11d). In comparison, the tidal tail t-S has a velocity gradient in the NW-SE direction (Sect. 5.3). The velocity gradient of $H 31 \mathrm{G}$ is $\sim 80 \mathrm{~km} \mathrm{~s}^{-1}$ with a PA of $90^{\circ}$ (Fig. 9d). The inclination derived from the optical isophotes yields a deprojected amplitude of $190 \mathrm{~km} \mathrm{~s}^{-1}$. This value is somewhat large for this galaxy according to the Tully-Fisher relation as given by Pierce \& Tully (1992), but this is not surprising since the association of $\mathrm{H} 31 \mathrm{G}$ with the prominent $\mathrm{t}-\mathrm{S}$ tidal tail will certainly produce a strong perturbation in the HI kinematics.

\section{4. $H 31 Q$}

H31Q has been classified as an elliptical by R90 based on its appearance on the POSS images. Our new optical and HI data (Fig. 12) show it to be a spiral galaxy with two distinct morphological components. At small radii $\left(<10^{\prime \prime}\right)$ the $B$ band isophotes are elongated $(b / a \sim 0.65)$ and asymmetrical, extending farther to the NE, twisting from a PA of $60^{\circ}$ to $44^{\circ}$ (Fig. 12a). The inner structure is enhanced in the median filter sharpened image (Fig. 12b). An elongated feature $\left(2 .{ }^{\prime} 5 \times 11^{\prime \prime}\right)$ bending toward the NE is seen, and this may indicate that H31Q has a small stellar bar, that would require a NIR image for a confirmation of its reality. At larger radii the isophotes become rounder, reaching an axial ratio of 0.95 , and their position angle goes up to $70^{\circ}$. Several weak knots are located at the west side of the galaxy. The surface brightness profile of this galaxy shows an exponential law corresponding to a disk (Fig. 13a). Furthermore the optical spectrum (Fig. 13b) shows spatially extended $\mathrm{H} \alpha$ emission, shifted with respect to the continuum center of the galaxy by $2 ., 5$ to the NE (along with [SII] lines) while $[\mathrm{NII}]$ is barely detected, indicating recent star formation and low metallicity.

The HI emission in H31Q is well centered on the optical peak (Fig. 12c), is detected from $V=4053$ to $4138 \mathrm{~km} \mathrm{~s}^{-1}$ and connects in the outer parts with t-NE and t-NW (Figs. 3 and 4). The integrated spectrum is shown in Fig. 5f. Its kinematics is characteristic of rotation with a kinematical major axis of PA $=50^{\circ}$ (Fig. 12d). The corresponding position velocity cut (Fig. 9e) has a rotational amplitude of $45 \mathrm{~km} \mathrm{~s}^{-1}$, with its kinematical center located at $4095 \mathrm{~km} \mathrm{~s}^{-1}$. The velocity range is larger along a $\mathrm{PA}=70^{\circ}\left(115 \mathrm{~km} \mathrm{~s}^{-1}\right)$, since $\mathrm{H} 31 \mathrm{Q}$ connects smoothly with t-NW along this direction for radii larger than $\sim 15^{\prime \prime}$. This is shown in a position velocity cut along the dashed line marked in Fig. 12d (see Fig. 9f).

\section{5. $A 0500-0434$}

This galaxy located $15^{\prime}$ (239 kpc) south of the group center previously had no known redshift. Our HI data clearly show it to be at the same redshift as the group $\left(V=3952 \mathrm{~km} \mathrm{~s}^{-1}\right)$. This HI system was not noticed by W91 since the emission (spreads over about $230 \mathrm{~km} \mathrm{~s}^{-1}$ in velocity $V=3852-4053 \mathrm{~km} \mathrm{~s}^{-1}$ ) is below the noise level of W91 in most channels. The derived total $\mathrm{HI}$ mass is $1.1 \times 10^{9} M_{\odot}$. Its $\mathrm{R}_{25}$ size as estimated from the image is $13.8 \mathrm{kpc}$. The integrated $\mathrm{HI}$ image of this galaxy is shown superposed on our R image in Fig. 14a, and the channel maps are shown in Fig. 15. The HI is brighter to the north, and shows double-horned HI profile (Fig. $5 \mathrm{~g}$ ), consistently with a highly inclined disk $\left(i \sim 62^{\circ}\right.$, PA $\left.=20^{\circ}\right)$. The inner disk is relatively devoid of $\mathrm{HI}$, reaching only $40 \%$ of the peak column density. The velocity field (Fig. 14b) is consistent with regular disk rotation in the center and a hint of warp in the outer disk. The kinematical major axis $\left(\mathrm{PA}=22^{\circ}\right)$ is well aligned with the optical one. The derived rotation velocity is about $190 \mathrm{~km} \mathrm{~s}^{-1}$ centered at $3952 \mathrm{~km} \mathrm{~s}^{-1}$ (Fig. 9g).

\section{Intragroup material}

A rich and complex history of tidal interactions within HCG 31 is suggested by numerous tidal features, most prominently in HI. They are identified and labeled as t-E, t-S, t-NW, t-NE in Fig. $3 \mathrm{~b}$ and $\mathrm{t}-\mathrm{C}$ in Fig. $4\left(V=4095 \mathrm{~km} \mathrm{~s}^{-1}\right)$. Only $\mathrm{t}-\mathrm{S}$ is prominent in optical light as a string of bright knots $(\mathrm{H} 31 \mathrm{E}$ and F), while a faint tip exists north of t-NW. We discuss each tidal feature in some detail below.

\subsection{Northeastern tail ( $t-N E)$}

This tidal feature links H31A and H31Q and has a full extent of $35 \mathrm{kpc}, 4$ times larger than $\mathrm{H} 31 \mathrm{~A}$ and has a total HI mass of $3 \times 10^{8} M_{\odot}$. Its integrated total flux density is similar in the DnC and $\mathrm{CnB}$ configuration data (see Fig. 5h), and this is 


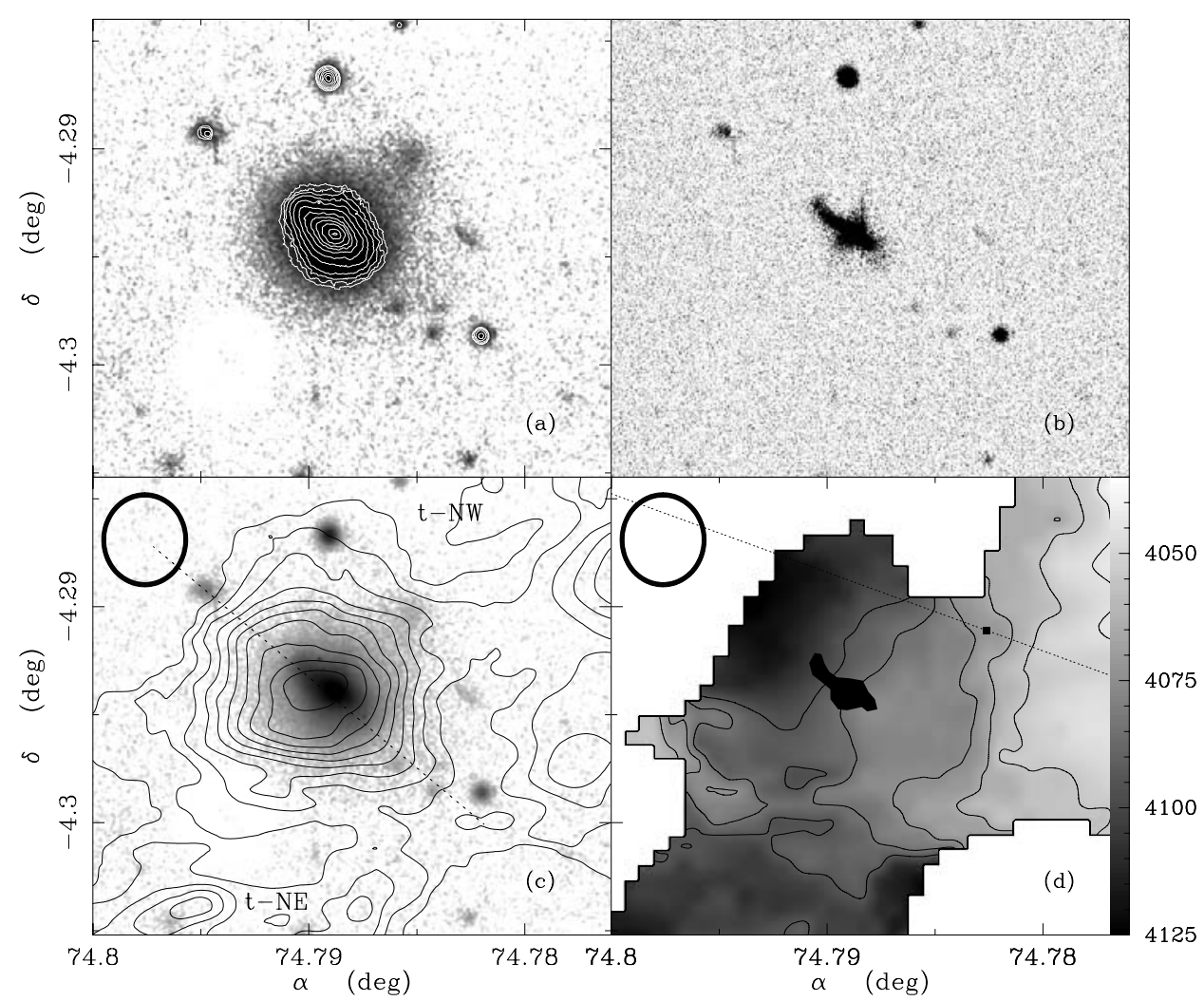

Fig. 12. a) $B$-band image of $\mathrm{H} 31 \mathrm{Q}$ in logarithmic gray-scale (saturated in order to show the low level features). The isophotal contours plotted in white are 21.1, 21.2, 21.6, 21.8, 22.1, 22.4, 22.8, 23.2, 23.6, 24.0, 24.2 and $24.4 \mathrm{mag} \mathrm{arcsec}^{-2}$. b) A sharpened $B$-band image of H31Q obtained by subtracting a $4^{\prime \prime} \times 4^{\prime \prime}$ median filtered image. c) The CnB-array velocity integrated $\mathrm{HI}$ image of $\mathrm{H} 31 \mathrm{Q}\left(V=4053-4138 \mathrm{~km} \mathrm{~s}{ }^{-1}\right)$. The column density contours are $0.5,1.4,2.4,3.3,4.2,5.2,6.1,7.1,8.0$ and $9.0 \times 10^{20}$ atoms $\mathrm{cm}^{-2}$ and have been overlapped on a B image of $\mathrm{H} 31 \mathrm{Q}$ in a logarithmic gray-scale representation. The dotted line indicates the PA of $50^{\circ}$ used for the position velocity plot shown in Fig. 9e. The synthesized beam $\left(15 .{ }^{\prime \prime} 8 \times 14 .^{\prime \prime} 5\right)$ is plotted in the upper left. d) The first moment map of the radial velocity. Both iso-velocity contours and gray-scale are shown. The contours range between 4035 to $4125 \mathrm{~km} \mathrm{~s}^{-1}$ in steps of $5 \mathrm{~km} \mathrm{~s}^{-1}$. The dotted line indicates the PA of $70^{\circ}$ used for the position velocity plot shown in Fig. 9f. A sketch of the sharpened image shown in b) is plotted in black.

a good indication that emitting regions are clumpy and compact in nature. The emission is stronger at $4095 \mathrm{~km} \mathrm{~s}^{-1}$ and $4106 \mathrm{~km} \mathrm{~s}^{-1}$ and fainter at $4085 \mathrm{~km} \mathrm{~s}^{-1}$ and $4117 \mathrm{~km} \mathrm{~s}^{-1}$. It appears connected to the northern stellar extension of $\mathrm{H} 31 \mathrm{~A}$ (see Sect. 4.1, Fig. 8a) but has no obvious stellar counterpart.

\subsection{Eastern tail (t-E)}

This is a faint but large plume of atomic gas with no obvious optical counterpart. The western part of this feature forms an extension of the HI emission of H31A at the channel map (Fig. 4) centered at $4138 \mathrm{~km} \mathrm{~s}^{-1}$ and is visible to $4042 \mathrm{~km} \mathrm{~s}^{-1}$, $59 \mathrm{kpc}$ east of H31A. The total HI mass amounts to $90 \%$ of the actual atomic content of H31A. Although it connects smoothly to the disk rotation of $\mathrm{H} 31 \mathrm{~A}$, the $90 \mathrm{~km} \mathrm{~s}^{-1}$ velocity gradient of this tail is opposite to the rotation of H31A (see Figs. 8b and $9 \mathrm{~h}$ ). If this feature is tidally driven originating from the outer disk of H31A, the orbital plane of the gas is strongly warped or the gas may be experiencing deceleration and infall back onto the galaxy. The channel maps in Fig. 4 with velocities between 4095 and $4138 \mathrm{~km} \mathrm{~s}^{-1}$ show a weak extension of this tail in the direction of $\mathrm{t}-\mathrm{S}$.

\subsection{Southern and central component ( $t-S$ and $t-C)$}

The southern tail (t-S) is the brightest and largest tidal feature, both in HI and starlight, joining the center of the group and $\mathrm{H} 31 \mathrm{G}$, extending for $56 \mathrm{kpc}$ (approximately 7 times the optical size of H31A; see Fig. 3a). Its HI content is $6.4 \times 10^{9} M_{\odot}$, comparable to the combined mass of $\mathrm{H} 31 \mathrm{~A}, \mathrm{H} 31 \mathrm{~B}$, and $\mathrm{H} 31 \mathrm{C}$ together. $\mathrm{HI}$ is found over a wide velocity range of nearly $200 \mathrm{~km} \mathrm{~s}^{-1}$ (Fig. 5j). This is one of the two locations among the extensive network of tidal features where the HI column density is higher than $10^{21}$ at $\mathrm{cm}^{-2}$, which is the empirical threshold for star formation (Skillman 1987). The other location is the northern clump in t-NW. Therefore it is not surprising to find here a prominent optical counterpart of a group of very blue knots, presumably the sites of ongoing massive star formation (Fig. 1).

Two clusters of optical knots, identified as features $\mathrm{E}$ and $\mathrm{F}$ in Fig. 1, are likely associated with the prominent $\mathrm{HI}$ tail $\mathrm{t}-\mathrm{S}$. The E knots are detected in the $I$-band image while the F knots are barely detected, and this may indicate that a subjacent stellar population exists in the E knots but not in the F knots. Knots $\mathrm{e} 1$ and $\mathrm{e} 2$ are connected by a luminous bridge with a PA of $20^{\circ}$, $\mathrm{e} 3$, e4, and e5, as well as $\mathrm{f} 1, \mathrm{f} 2$ and $\mathrm{f} 3$ are aligned with a PA 

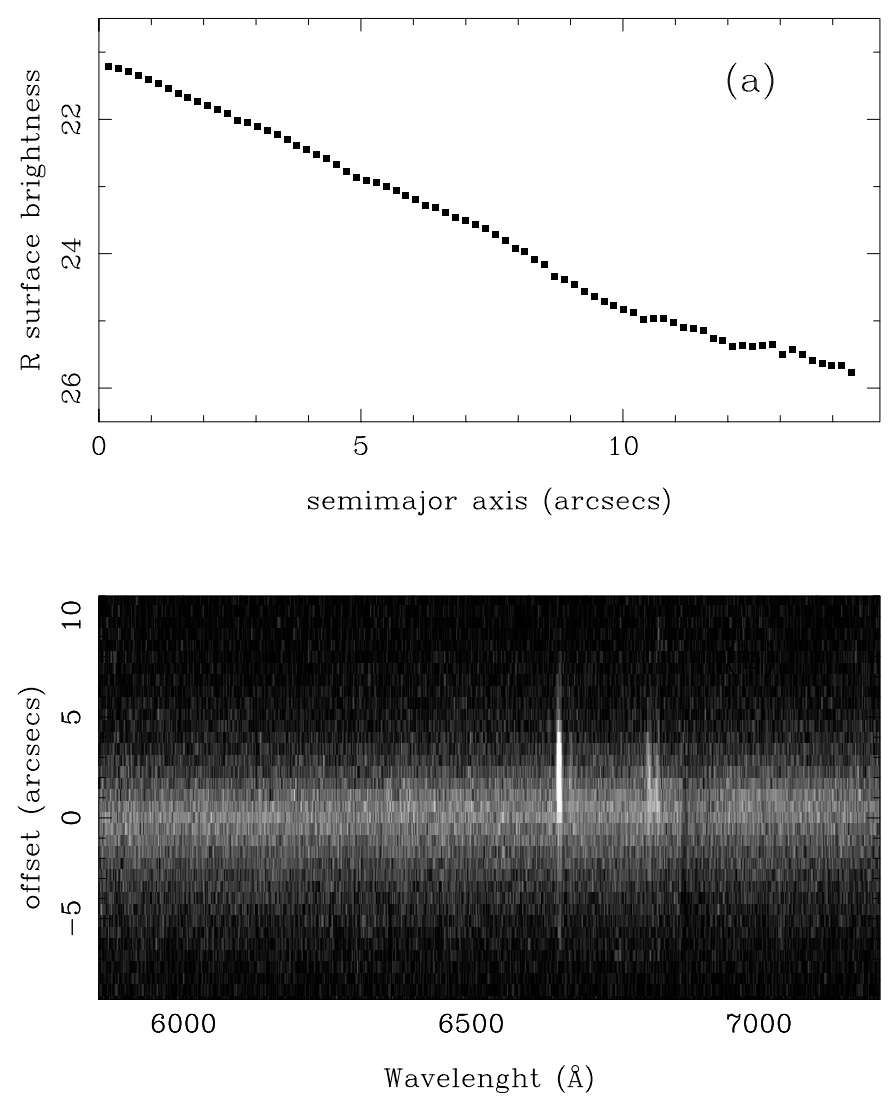

Fig. 13. a) Radial profile of the $R$-band light in H31Q as a function of the semi-major axis distance. b) A long-slit spectrum in the $\mathrm{H} \alpha$ region of $\mathrm{H} 31 \mathrm{Q}$ in the major axis direction. Spatial positions are given in offsets with respect to the continuum peak along the slit.
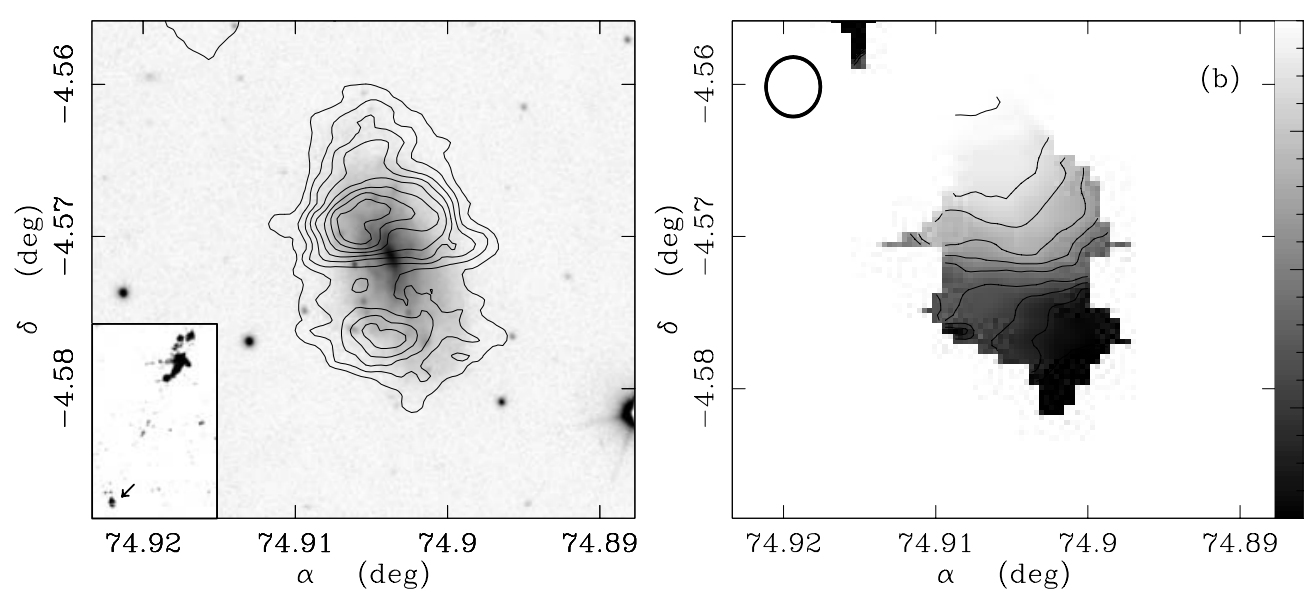

Fig. 14. a) The $\mathrm{CnB}$ array integrated $\mathrm{HI}$ image of the galaxy A0500-0434 $\left(V=3852-4053 \mathrm{~km} \mathrm{~s}^{-1}\right)$ in contours superimposed on the new NOT $R$-band image. The contours correspond to HI column densities of $0.9,1.9,2.8,3.8,4.7,5.7,6.6$, and $7.5 \times 10^{20} \mathrm{atoms} \mathrm{cm}^{-2}$. In the lower left panel we show a reduced gray-scale map of the HI emission of HCG 31 and its environment, and mark A0500-0434 with an arrow 15' south of HCG 31. b) The first moment image of the radial velocity field. The scale goes as in the wedge where the heliocentric velocities are indicated in $\mathrm{km} \mathrm{s}^{-1}$. The contours range between 3860 and $4040 \mathrm{~km} \mathrm{~s}^{-1}$ in steps of $10 \mathrm{~km} \mathrm{~s}^{-1}$. The beam size is 15 ." $8 \times 14$.' 5 .

of $42^{\circ}$, and f5, f6 and $\mathrm{f} 7$ with a PA of $56^{\circ}$. The origin of these alignments is not well understood.

The knots $\mathrm{f} 1$ and $\mathrm{f} 2$ are contained in an HI cloud with a velocity gradient of $\sim 70 \mathrm{~km} \mathrm{~s}^{-1}$ nearly perpendicular to the tail $\left(\mathrm{PA} \sim 52^{\circ}\right)$ suggestive of rotation (Fig. 16). The high resolution channel maps (Fig. 4) show the blueshifted tip of the tail located SE of $\mathrm{H} 31 \mathrm{G}$, between $V=3937$ and $3958 \mathrm{~km} \mathrm{~s}^{-1}$, reaching redder velocities from the NW part of $\mathrm{H} 31 \mathrm{G}$ to $\mathrm{H} 31 \mathrm{AC}$ area, from 3979 to $4074 \mathrm{~km} \mathrm{~s}^{-1}$. The extreme redshifted emission seems to be associated with several optical knots at different velocities (e3 - e5 from $V=4074$ to $4011 \mathrm{~km} \mathrm{~s}^{-1}$; f5 - f7 from 4000 to $4053 \mathrm{~km} \mathrm{~s}^{-1}$; f3 and f4 from $V=3989$ to $4032 \mathrm{~km} \mathrm{~s}^{-1}$ ). It merges with the tidal tail $\mathrm{t}-\mathrm{C}$, which is a $8 \mathrm{kpc}$ bridge joining H31 A and B. This bridge overlaps with e1 and $\mathrm{e} 2$ in the channel maps, extending over a velocity range of $\sim 50 \mathrm{~km} \mathrm{~s}^{-1}$. 


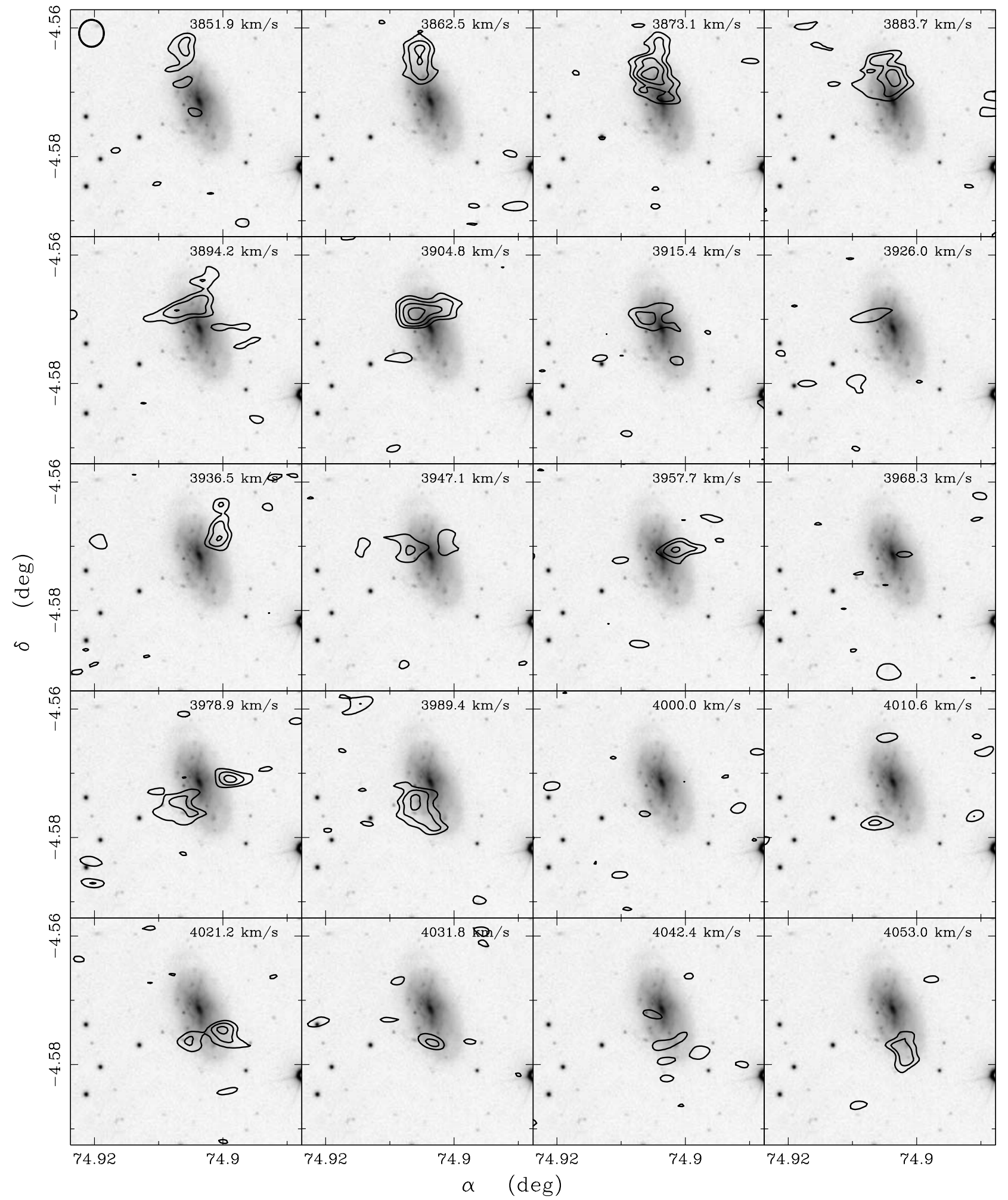

Fig. 15. Channel maps of the $21 \mathrm{~cm}$ line radiation in the galaxy A0500-0434 superimposed on the new NOT $R$-band image. Heliocentric velocities are indicated in each panel. Contours correspond to $-3.0,3.0,4.3,5.8$ and $7.4 \mathrm{~K}(1 \sigma=1.2 \mathrm{~K})$. The synthesized beam $\left(15\right.$." $^{\prime} 8 \times 14$." $\left.^{\prime}\right)$ is plotted in the upper left panel.

\subsection{Northwestern tail (t-NW)}

This $50 \mathrm{kpc}$ long, massive $\left(M(\mathrm{HI})=4 \times 10^{9} M_{\odot}\right.$; Fig. $\left.5 \mathrm{k}\right) \mathrm{HI}$ tail is made of extended material better seen in the low resolution DnC array map (Fig. 3). It appears to connect the group center with a compact clump located on the western part of H31Q (Sect. 4.4) and a second clump with a weak optical counterpart at the northern tip. The column density at this clump is $10^{21} \mathrm{at} \mathrm{cm}^{-2}$, which is one of the highest seen among the tidal filaments. The channel maps show that the southern part of the 


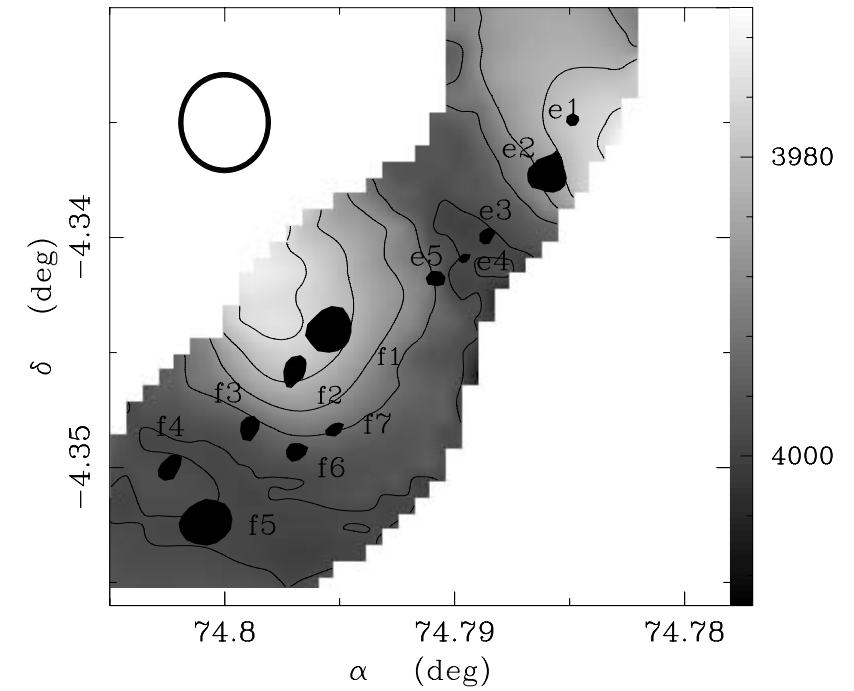

Fig. 16. The first moment image of the radial velocity field in and around H31F. Both iso-velocity contours and gray-scale are shown. The contours range between 3970 to $4030 \mathrm{~km} \mathrm{~s}^{-1}$ in steps of $10 \mathrm{~km} \mathrm{~s}^{-1}$. The beam size is 15 ." $^{\prime} 8 \times 14$." $^{\prime}$ and is plotted in the upper left. The optical knots are sketched in black.

tail could well be the tidal debris from the NE side of the disk of $\mathrm{H} 31 \mathrm{~B}$, but we cannot rule out its origin in H31AC.

\section{Discussion}

\subsection{Neutral gas content and star formation}

In VM01 we have shown that the total HI content of HCG 31 as a group is normal for the optical luminosity and morphology of its members. On the other hand, if only the gas found within the main bodies of the galaxies is considered, individual members are deficient in $\mathrm{HI}$; the $\mathrm{HI}$ content of the galaxies in the group range from 10 to $83 \%$ of their normal expected values (see Table 3 in VM01). Although HI is detected in all of the individual members, $60 \%$ is located in 4 tidal tails and 1 bridge that link the 5 galaxies. Yun et al. (1997) and Verdes-Montenegro et al. (1998) also found the molecular mass of H31 A, C and B to be low for their optical luminosities. In H31A and H31C this might be attributed to the enhancement of the $B$-band luminosity resulting from ongoing massive star formation activity and/or their $\mathrm{CO}$ emission being underluminous because of low metallicity (R90; Richer et al. 2003; Lopez-Sanchez et al. 2004). Tidal stripping might have also affected the molecular gas since $\mathrm{CO}$ emission is detected only on one side of the galaxy in $\mathrm{H} 31 \mathrm{~B}$.

The current star formation activity is concentrated in $\mathrm{H} 31 \mathrm{~A}$ and $\mathrm{C}, \mathrm{H} 31 \mathrm{G}$ and $\mathrm{H} 31 \mathrm{~F}$, traced by the blue optical color and radio continuum emission (Fig. 6). Both large gas mass and high mean density are needed to fuel massive star formation (e.g., Kennicutt 1998). In HCG 31 the highest column density of neutral gas is found in the intersection of $\mathrm{H} 31 \mathrm{~A}$ and $\mathrm{C}$, as well as in $\mathrm{H} 31 \mathrm{~F}$ with $3 \times 10^{21}$ at $\mathrm{cm}^{-2}$ and in $\mathrm{H} 31 \mathrm{G}$ with $2 \times$ $10^{21}$ at $\mathrm{cm}^{-2}$ (see Fig. 3). Naturally these are also the sites of the current star forming activity.

\subsection{A tidal dwarf in formation}

The bulk of the HI tidal material $\left(6.4 \times 10^{9} M_{\odot}\right)$ is located in the southern tail t-S. About $10 \%$ of this material is kinematically detached from the tail, clearly showing a velocity gradient of $70 \mathrm{~km} \mathrm{~s}^{-1}$ nearly perpendicular to that of the tail (Sect. 5.3). The blue optical knots f1 and f2 (Figs. 1 and 3c) are found here, and the peak HI column density reaches $3 \times 10^{21}$ at $\mathrm{cm}^{-2}$. Furthermore the optical colors of $\mathrm{H} 31 \mathrm{~F}$ do not indicate the presence of a subjacent population in this knot. These results support the idea that the stars in $\mathrm{H} 31 \mathrm{~F}$ have formed within the tail, making of this dynamically decoupled clump an excellent candidate for a tidal dwarf in formation.

\subsection{Galaxy evolution in the compact group environment}

Unlike a textbook example of two interacting galaxies with two prominent tidal tails (e.g. Hibbard \& van Gorkom 1997; Hibbard \& Yun 1999), HCG 31 is composed of 5 galaxies and 5 tidal features. Consequently interpreting the detailed tidal history of this system is extremely difficult. Our approach is to focus our attention on the most relevant perturbations found in the individual galaxies as well as the gas mass and morphology of the tidal tails.

All member galaxies of HCG 31 show signs of interactions. Although gas and stars are heavily perturbed in $\mathrm{H} 31 \mathrm{AC}$, including a warp in $\mathrm{H} 31 \mathrm{~A}$, we conclude from our data that it is not in a late stage merger as suggested by W91 and others because their HI components and velocity fields can be still be separated relatively cleanly (see Sect. 4.1). The overlap of the two rotating disks along the line of sight is producing the high velocity dispersion found by Richer et al. (2000) along a ridge between $\mathrm{H} 31 \mathrm{~A}$ and $\mathrm{C}$. Our $B-I$ color image shows a wide red strip along H31A that suggests that this galaxy is in front of H31C, dimming its light in the overlapping area. In H31B, both the optical and HI emission are lopsided and a shift exists between the HI with respect to the stellar light distribution in the southern half of the galaxy. It has been suggested that this galaxy has a warped optical disk (see O'Halloran et al. 2002, and references therein). However the observed morphology may be better explained by projected open spiral arms, tidal in origin. Briggs (1990) found that warps typically occur at radii larger than $R_{H_{0}}\left(\sim 30^{\prime \prime}\right.$ for H31B), but the twist seen here is found at $\sim 7^{\prime \prime}$. Similar spiral features are in galaxies like NGC 3550 and NGC 4731, and the spiral pattern in NGC 4731 has been explained as induced by a tidal interaction with its companion (Sandage \& Bedke 1995). In addition, a type II surface brightness profile points toward a galaxy with morphology modified by interaction. This evidence is not restricted to any particular inclination range and it cannot be explained by an extinction effect as shown by different authors (MacArthur et al. 2003, 2004; Anderson et al. 2004). Some tentative models exist that might explain this type of profile. One plausible explanation involves a radial redistribution of central stars into a ring through barlike perturbations (MacArthur et al. 2003), however a one to one relation between type-II profiles and barred structure in galaxies has not been established. An alternative 
Table 4. Possible origins of the Tidal Tails and the galaxy HI contents.

\begin{tabular}{cccccc}
\hline \hline Galaxy & Tail & $\begin{array}{c}\log M(\mathrm{HI})_{\text {gal }} \\
\left(M_{\odot}\right)\end{array}$ & $\begin{array}{c}\log M(\mathrm{HI})_{\text {tail }} \\
\left(M_{\odot}\right)\end{array}$ & $\begin{array}{c}\log M(\mathrm{HI})_{\text {pred }}^{1} \\
\left(M_{\odot}\right)\end{array}$ & $\begin{array}{c}\log M(\mathrm{HI})_{\text {sum }}^{2} \\
\left(M_{\odot}\right)\end{array}$ \\
\hline H31A & t-E & 9.30 & 9.41 & 9.54 & 9.66 \\
H31B & t-NW & 9.28 & 9.59 & 9.71 & 9.76 \\
H31C & t-S & 9.20 & 9.81 & 10.32 & 9.91 \\
\hline
\end{tabular}

1 The uncertainty in $M(\mathrm{HI})_{\text {pred }}$ is about a factor of 2 (or 0.3 in dex). See VM01 for a detailed discussion.

$2 M(\mathrm{HI})_{\text {sum }}$ is the combined HI mass between the galaxy and the identified tidal tail. This is to be compared with $M(\mathrm{HI})_{\text {pred }}$.

explanation involves the existence of an inner truncated disk that might be the result of processes that inhibit star formation in that region (Anderson et al. 2004) and produced by resonances associated with the disk kinematics. The above authors pointed out that barlike perturbing potentials or a lopsided mass distribution are possible mechanisms to drive such resonances. Our observed profiles may be explained by the last scenario. Also Marquez and Moles $(1996,1999)$ found that type II profiles occur only in interacting pairs in a surface photometry comparison study between samples of well isolated spirals and spirals in pairs.

The HI filaments found in HCG 31 offer a unique clue on the group evolution. Their location and gas mass contents are indicators of their parent galaxies, and their physical extents and morphology reflect the dynamical history of the interactions involved. Since we deduced that there is no missing $\mathrm{HI}$ in HCG 31 as a group, the missing HI from the individual galaxies must be located in the tidal features. The scatter in the relationship between HI mass and luminosity is large (see Haynes \& Giovanelli 1984, VM01), so that the conclusions given below have to be taken as indicative but not definitive of the tail formation history.

Possible matches of the individual galaxies and the tidal features derived from the kinematics and morphology are summarized in Table 4. Since t-E appears to link physically only with $\mathrm{H} 31 \mathrm{~A}$, it is natural to assign its origin to $\mathrm{H} 31 \mathrm{~A}$. When the $\mathrm{HI}$ masses of t-E and H31A are added, the sum is close to the normal HI content for H31A (Table 4). The projected proximity between $\mathrm{H} 31 \mathrm{~A}$ and $\mathrm{C}$ suggests that the interaction between H31A and C stripped t-E from H31A. Similarly H31C is clearly deficient in HI, and only t-S has a comparable HI mass and the bulk of its emission linked with $\mathrm{H} 31 \mathrm{C}$. When the HI masses of $\mathrm{H} 31 \mathrm{C}$ and t-S are combined, again the total HI matches the expected $\mathrm{HI}$ mass for $\mathrm{H} 31 \mathrm{C}$ (Table 4). Hence we can reasonably conclude that $\mathrm{H} 31 \mathrm{C}$ has significantly contributed to this long tail $\mathrm{t}-\mathrm{S}$. The location of $\mathrm{H} 31 \mathrm{G}$ in $\mathrm{t}-\mathrm{S}$ indicates that it played a significant role in producing the main tidal feature $\mathrm{t}-\mathrm{S}$. H31B is also deficient in $\mathrm{HI}$, and the low resolution $\mathrm{HI}$ image shown in Fig. 3 a suggests the tidal feature t-NW originating from the general vicinity of $\mathrm{H} 31 \mathrm{~B}$. Adding the $\mathrm{HI}$ mass of $\mathrm{t}-\mathrm{NW}$ to $\mathrm{H} 31 \mathrm{~B}$ restores its $\mathrm{HI}$ content to the normal level as summarized in Table 4. H31Q sits in the middle of the brightest HI peak in t-NE while the HI peak in t-NE also has a faint optical counterpart. There is a possible bridge of $\mathrm{HI}$ connecting the two HI features, it is suggestive that H31Q played a role in
Table 5. Size, velocity semiamplitudes ${ }^{1}$ and masses of HCG 31 galaxies.

\begin{tabular}{crrc}
\hline \hline Gal & $\begin{array}{c}R \\
(\mathrm{Kpc})\end{array}$ & $\begin{array}{c}\Delta v^{1} \\
\left(\mathrm{~km} \mathrm{~s}^{-1}\right)\end{array}$ & $\begin{array}{c}M \\
\left(10^{9} M_{\odot}\right)\end{array}$ \\
\hline H31A & 3.87 & 90.0 & 7.3 \\
H31B & 4.70 & 47.2 & 2.4 \\
H31C & 2.17 & 39.8 & 0.8 \\
H31G & 3.93 & 95.0 & 8.3 \\
H31Q & 3.63 & 36.9 & 1.2 \\
\hline A0500-0434 & 7.46 & 109.5 & 20.8 \\
\hline
\end{tabular}

1 We list the semiamplitudes of the velocity deprojected from inclination.

forming both of these features and possibly in the tidal disruption of H31B. These three main galaxies and their respective tidal features account for $97 \%$ of the tidal material in HCG 31 . Given the complexity of this system, including projection effects, these suggested interaction scenarios are only plausible at best.

The physical extents of these tidal features are similar in size to those found among other strongly interacting systems such as the Toomre Sequence studied by Hibbard \& Van Gorkom (1997). Their projected sizes range between 35 and $60 \mathrm{kpc}$, which are somewhat smaller than the average for the Toomre Sequence. On the other hand, HCG 31 galaxies have typically one half of the optical sizes of the Toomre Sequence mergers, and the ratio of the tail lengths to optical diameter (a factor 5-7) falls among the largest of in the Toomre Sequence. The presence of those tidal features implies that collisions have taken place with a small impact parameter, but not so low to produce a merger, which is compatible with the low intergalactic separation observed in this group.

We obtained estimations for the individual masses of the galaxies of the group following Burstein \& Rubin (1985). For each member we evaluated $m_{i} \propto\left(\Delta v_{i}\right) 2 R_{i}$ where we adopted for $\Delta v$ the value of the semiamplitude of the HI rotation curve and the corresponding HI radius. Both quantities and the individual mass estimations are presented in Table 5. All the galaxies correspond to small low mass late type systems. An estimation of the total dynamical mass of HCG 31 can be obtained from the central positions and the HI velocities of the group galaxies. Under the assumptions that light traces mass, dynamical relaxation and spherical symmetry, the virial $M_{v}$ and 
Table 6. Dynamical parameters of HCG 31.

\begin{tabular}{lcc}
\hline \hline & Unweighted & Weighted \\
\hline$\langle v\rangle\left(\mathrm{km} \mathrm{s}^{-1}\right)$ & 4057 & 4077 \\
$\sigma_{v}\left(\mathrm{~km} \mathrm{~s}^{-1}\right)$ & $67 \pm 6$ & $61 \pm 9$ \\
$R_{\mathrm{h}}(\mathrm{Kpc})$ & $20 \pm 9$ & $32 \pm 13$ \\
$M_{v}\left(10^{11} M_{\odot}\right)$ & $1.9 \pm 1$ & $3 \pm 2$ \\
$M_{\mathrm{p}}\left(10^{11} M_{\odot}\right)$ & $2 \pm 0.5$ & $3 \pm 1$ \\
\hline
\end{tabular}

projected mass $M_{\mathrm{p}}$ estimators provide a measurement of the total (dark+visible) mass of the group (see Heisler et al. 1985; Perea et al. 1990),

$M_{v}=\frac{3 \pi}{G} \sigma_{v} 2 R_{\mathrm{h}}, \quad$ and $\quad M_{\mathrm{p}}=\frac{32}{\pi G}\left(\frac{4-2 \beta}{4-3 \beta}\right)\left\langle v^{2} R\right\rangle$

where the virial mass is defined in terms of the mean harmonic radius, $R_{\mathrm{h}} \equiv\left(2 / \sum_{i} m_{i}^{2}\right) \times \sum_{i, j} m_{i} m_{j} / R_{i j}$, and the velocity dispersion, $\sigma_{v}^{2} \equiv \sum_{i} m_{i} v_{i} 2 / \sum_{i} m_{i}$. The parameter $\beta$ appearing in $M_{\mathrm{p}}$ depends of the nature the orbits of the system and its value is zero for total isotropy.

The $M_{v}$ and $M_{\mathrm{p}}$ estimations are given in Table 6 , where we also include both estimators without weighting by mass. Quoted uncertainties were estimated using the Jackknife method $^{2}$. A0500-0434 is excluded in this calculation since it is clearly distinguished from the rest of the group in projected spatial distribution as discussed further below. The crossing time of the group $\left(t_{\mathrm{CR}}=(\pi / \sqrt{3}) \times R_{\mathrm{h}} / \sigma_{v}\right)$ is found to be quite short $\left(0.05 \mathrm{H}_{0}^{-1}\right)$, suggesting that the group is already dynamically relaxed and allow us for applying both mass estimators. The total dynamical mass of the group amounts to about $2 \times 10^{11} M_{\odot}$, whereas the sum of the individual masses is $2 \times 10^{10} M_{\odot}$. This fact suggests the presence of a common dark halo with a mass a few times larger than the sum of the masses of the individual galaxies, in agreement with the models described by Athanassoula et al. (1997) and Perea et al. (2000).

The total dynamical mass places some useful constraints on the characteristics of the two large tidal plumes. At the projected distance of the $\mathrm{t}-\mathrm{S}$ plume, the escape velocity $(\sqrt{2 G M / R})$ is about $200 \mathrm{~km} \mathrm{~s}^{-1}$ whereas the velocity of the plume with respect to the projected center of mass is about $100 \mathrm{~km} \mathrm{~s}^{-1}$. This means that an inclination angle has to be larger than $60^{\circ}$ for the plume material to escape from the group. The situation is similar for the t-E plume which has similar physical properties as the t-S plume. Therefore, both tails will likely fall back onto the group again eventually and settle around the group similar to those seen in H49.

The newly identified neighbor A0500-0434 has a mass comparable to the total dynamical mass of HCG 31 as a group (see Table 5). If included as a member of the group, the group mass estimates increase to $M_{v}=(3 \pm 1) \times 10^{11} M_{\odot}$ and $M_{\mathrm{p}}=(7 \pm 2) \times 10^{11} M_{\odot}$, depending on the weighting. The

\footnotetext{
2 Jacknife algorithm estimates the variance in the statistical quantity by resampling the original sample by eliminating one of the values or cases successively.
}

velocity difference of A0500-0434 with respect to the group is low $\left(76 \mathrm{~km} \mathrm{~s}^{-1}\right)$ while its escape velocity from the group center is $85 \mathrm{~km} \mathrm{~s}^{-1}$. Given the large uncertainty in the projection angle for the orbit, whether this galaxy is a gravitationally bound system to the group is highly uncertain. There is little observational evidence supporting any recent interaction involving A0500-0434 with the rest of the group, and we conclude that it does not play now an important role in the evolution of HCG 31 as a group.

\subsection{The future of HCG 31}

By analyzed the total HI content of 72 HCGs and the detailed spatial distribution and kinematics of HI within a subset of 16 groups using VLA HI data, we have established that HCGs have on average about $40 \%$ of the expected HI for their optical luminosity and morphological types of the member galaxies (see VM01). The HI deficiency is even larger for the individual galaxies ( $24 \%$ of the expected $\mathrm{HI}$ ), and the numerous $\mathrm{HI}$ tidal features seen in the VLA HI images suggest that efficient gas stripping into the group environment is an important reason for the definciency. Based on these observations, we have proposed a three phase evolutionary scenario for compact groups. During the phase 1 the groups show a low level of interaction and most of the $\mathrm{HI}$ is found within the galaxies, and tidal disruption of individual member galaxies is not yet important. During the phase 2 multiple tidal tails form so that a large amount of the atomic gas is found in the intragroup medium. Once the gas is removed from the individual galaxies, the evolution may lead to two different paths. In one evolutionary path (phase 3a) tidally removed gas is destroyed, producing HI deficient groups (e.g. HCG 92; VM01; Sulentic et al. 2001; Williams et al. 2002). Groups composed of small galaxies seems to follow another path (phase 3b) where tidally removed gas forms a single cloud (e.g. HCG 49; VM01). These disparate conclusions may be dictated by the nature of the group potential and of the member galaxies as the heating of the tidally removed gas may be strongly dependent on the detailed dynamics within the group.

The present status of HCG 31 is best described as phase 2 since the largest ratio between the external $\mathrm{HI}$ to the disk $\mathrm{HI}$ in mass is expected during this stage (e.g., HCG 16, HCG 96). Since the intragroup medium accounts for the majority of the total HI mass within the group, HCG 31 is near the end of the phase 2 evolution and is about to enter the final evolutionary phase. The classic phase 3a group is HCG 92 where little HI is directly associated with the individual member galaxies and the group as a whole is highly deficient in HI content (VM01; Williams et al. 2002). In contrast, HCG 31 is not HI deficient as a group, and it resembles more closely the classic phase $3 \mathrm{~b}$ object HCG 49 (Verdes-Montenegro et al. in prep.; also see Fig. 7 in VM01). Both HCG 31 and HCG 49 consist of galaxies with low luminosity and small optical sizes $\left(R_{25} \sim 6 \mathrm{kpc}\right)$ and small median separations relative to their sizes. These characteristics likely result in the intense HI stripping already seen in HCG 31. HCG 31 and HGC 49 also share in common a low group velocity dispersion $\left(\sim 60 \mathrm{~km} \mathrm{~s}^{-1}\right.$ and $34 \mathrm{~km} \mathrm{~s}^{-1}$ for HCG 31 and 
HCG 49 respectively) while their observed HI line widths are larger than $200 \mathrm{~km} \mathrm{~s}^{-1}$. We conclude that HCG 31 will likely evolve into Phase $3 \mathrm{~b}$ where the tidally stripped gas form a common $\mathrm{HI}$ envelope containing $\mathrm{H} 31 \mathrm{~A}, \mathrm{~B}, \mathrm{C}, \mathrm{G}$ and $\mathrm{Q}$.

\section{Conclusions}

We have obtained new optical imaging and spectroscopic data and new VLA HI observations of a well studied Hickson Compact Group HCG 31. From the combined analysis of these multi-wavelength data, we obtained the following results:

- The HI distribution and velocity fields for H31A and H31C are shown to be distinct by our new high resolution VLA HI data with almost orthogonal orientations. Our new optical images suggest that $\mathrm{H} 31 \mathrm{~A}$ is located in front of $\mathrm{H} 31 \mathrm{C}$. Based on these results, we conclude that $\mathrm{H} 31 \mathrm{~A}$ and $\mathrm{H} 31 \mathrm{C}$ are not an advanced merger system.

- H31B shows indications of morphological and kinematical perturbations such as a lopsided emission in both $\mathrm{HI}$ and in the optical, a type II surface brightness profile with open projected spiral arms, and a positional shift between the HI and optical distributions.

- We have been able to separate the HI component of H31G from the southern tidal tail (t-S). The new data shows clear evidence for rotation in the E-W direction.

- We have identified a new companion of HCG 31, the spiral galaxy A0500-0434, using the VLA HI data at $240 \mathrm{kpc}$ south of the group center. Despite its proximity and large mass, there is little evidence that this galaxy interacted with members of HCG 31 in the recent past. We also establish clearly that $\mathrm{H} 31 \mathrm{Q}$, which has a spiral morphology, is a member of the group closely interacting with the rest of HCG 31.

- The total HI content of HCG 31 as a group is normal, but $60 \%$ of the $\mathrm{HI}$ is located in 4 tidal tails and 1 bridge that link the 5 individual galaxies making up the group. Intense stripping of gas and rapid evolution of the member galaxies is currently under way.

- The highest star formation activities are seen at the locations with the highest gas column density: H31AC, H31F, and H31G. Only those tails where the column density exceeds the $10^{21}$ at $\mathrm{cm}^{-2}$ threshold show a stellar counterpart (H31F and t-NW).

- The tidal tail with the highest HI content, t-S, has a decoupled HI clump $(\mathrm{H} 31 \mathrm{~F})$ with a transverse change in velocity of $70 \mathrm{~km} \mathrm{~s}^{-1}$, an HI mass of $6 \times 10^{8} M_{\odot}$ and a column density of $3 \times 10^{21}$ at $\mathrm{cm}^{-2}$. All these characteristics support it as a good candidate for a forming tidal dwarf.

- From the kinematics and morphology of the HI tidal features, we have deduced the origin of the tidal features $\mathrm{t}-\mathrm{E}$ and $\mathrm{t}-\mathrm{S}$ to be $\mathrm{H} 31 \mathrm{~A}$ and $\mathrm{H} 31 \mathrm{C}$, respectively. $\mathrm{H} 31 \mathrm{G}$ was involved in removing a significant amount of $\mathrm{HI}$ from $\mathrm{H} 31 \mathrm{C}$, forming $\mathrm{t}-\mathrm{S}$. The interaction between $\mathrm{H} 31 \mathrm{Q}$ and $\mathrm{H} 31 \mathrm{~B}$ likely has contributed to the formation of $\mathrm{t}-\mathrm{NW}$ and $\mathrm{t}-\mathrm{NE}$. These individual galaxies are deficient in $\mathrm{HI}$ mass for their intrinsic Hubble type and $B$-band luminosity, and adding the HI mass from the respective tidal tails restore their HI contents to normal levels.

- The dynamical mass of the group is consistent with the existence of a dark halo of a few times the mass measured in the galaxies, while $\mathrm{t}-\mathrm{S}$ and $\mathrm{t}-\mathrm{E}$ have high probabilities of being bound.

- The likely eventual fate of HCG 31 is to evolve to form a group of low luminosity galaxies surrounded by a common HI cloud envelope (Phase 3b, VM01), similar to HCG 49.

Acknowledgements. L.V.-M., A.O. and J.P. are partially supported by DGI (Spain) Grant AYA 2002-03338, AYA 2003-128 and Junta de Andalucía TIC-114 (Spain).

\section{References}

Anderson, K. S. J., Baggett, S. M., \& Baggett, W. 2004, AJ, 127, 2085 Athanassoula, E., Makino, J., \& Bosma, A. 1997, MNRAS, 286, 825 Bessell, M. S. 1983, PASP, 95, 480

Bessell, M. S. 1995, PASP, 107, 672

Briggs, F. H. 1990, ApJ, 352, 15

Burstein, D., \& Heiles, C. 1984, ApJS, 54, 33

de Carvalho, R. R., Ribeiro, A. L. B., \& Zepf, S. E. 1994, ApJS, 93, 47

Conti, P. S. 1991, ApJ, 377, 115

Dubinsky, J., Mihos, J. C., \& Hernquist, L. 1996, ApJ, 462, 576

Haynes, M. P., \& Giovanelli, R. 1984, AJ, 89, 758

Heisler, J., Tremaine, S., \& Bahcall, J. N. 1985, ApJ, 298, 8

Hibbard, J. E., \& van Gorkom, J. H. 1997, AJ, 111, 655

Hibbard, J. E., \& Yun, M. S. 1999, AJ, 118, 162

Hickson, P. 1982, ApJ, 255, 382

Hickson, P. 1993, ApL\&C, 29, 1

Hickson, P., Mendes de Oliveira, C., Huchra, J. P., \& Palumbo, G. G. C. 1992, ApJ, 399, 353

Huchra, J. P., Geller, M. J., Clemens, C. M., Tokaiz, S. P., \& Michel, A. 1993, Harvard-Smithsonian Center for Astrophysics

Iglesias-Páramo, J., \& Vílchez, J. M. 1997, ApJ, 479, 190

Johnson, K. E., Vacca, W. D., Leitherer, C., Conti, P. S., \& Lipsey, S. J. 1999, AJ, 117, 1708

Johnson, K. E., \& Conti, P. S. 2000, AJ, 119, 2146

Kennicutt, R. C. 1998, ARA\&A, 36, 189

Landolt, A. U. 1983, AJ, 88, 439

Landolt, A. U. 1992, AJ, 104, 340

Lopez-Sanchez, A. R., Esteban, \& Rodriguez, M. 2004, ApJS, 153, 243

MacArthur, L. A., Courteau, S., \& Holtzman, J. A. 2003, ApJ, 582, 689

MacArthur, L. A., Courteau, S., Bell, E., \& Holtzman, J. A. 2004, ApJS, in press [arXiv: astro-ph/0401437]

Márquez, I., \& Moles, M. 1996, A\&AS, 120, 1

Márquez, I., \& Moles, M. 1999, A\&A, 344, 421

O'Halloran, B., Metcalfe, L., McBreeb, B., et al. 2002, ApJ, 575, 747

Perea, J., del Olmo, A., \& Moles, M. 1990, A\&A, 237, 319

Perea, J., del Olmo, A., Verdes-Montenegro, L., et al. 2000, Small Galaxy Groups, ed. M. Valtonen, \& C. Flynn, IAU Coll., 174, 377

Pierce, M. J., \& Tully, R. B. 1992, AJ, 387, 47

Richer, M. G., Georgiev, L., Rosado, M., et al. 2003, A\&A, 397, 99

Rubin, V. C., Ford, W., Kent, Jr., \& Hunter, D. A. 1990, ApJ, 365, 86 (R90) 
Sandage, A., \& Bedke, J. 1994, The Carnegie atlas of galaxies, Vol. 1, Williams, B. A., Mc Mahon, P. M., \& Van Gorkom, J. H. 1991, AJ, Washington: the Carnegie Institution of Washington

Savage, B. D., \& Mathis J. S. 1979, ARA\&A, 17, 73

Skillman, E. D. 1987, in NASA Conf. Pub. 2466, Star Formation in Galaxies, ed. C. J. Lonsdale Persson (Washington: NASA), 263 101, 1957 (W91)

Williams, B. A., \& Rood, H. J. 1987, ApJS, 63, 265

Williams, B. A., Yun, M. S., \& Verdes-Montenegro, L. 2002, AJ, 123, 2417

Sulentic, J. W., Rosado, M., Dultzin-Hacyan, D., et al. 2001, AJ, 122, 2993

Young, A. T. 1974, ApJ, 189, 587

Yun, M. S., \& Carilli, C. L. 2002, ApJ, 568, 88

Verdes-Montenegro, L., Yun, M. S., Perea, J., Del Olmo, A., \& Ho, P. T. P. 1998, ApJ, 497, 89

Yun, M. S., \& Hibbard, J. E. 2001, ApJ, 550, 104

Yun, M. S., Reddy, N. A., \& Condon, J. J. 2001, ApJ, 554, 803

Verdes-Montenegro, L., Yun, M. S., Williams, B. A., et al. 2001, A\&A, 377, 812 (VM01)

Yun, M. S., Verdes-Montenegro, L., Del Olmo, A., Perea J. 1997, ApJ, 475, L21 